\title{
ENHANCED COUPLING AND DECOUPLING OF UNDERGROUND NUCLEAR EXPLOSIONS
}

\author{
R. W. Terhune \\ C. M. Snell \\ H, C. Rodean
}

September 4, 1979

\section{MASTER}

Work performed under the auspices of the U.S. Department of Energy by the UCLLL under contract number W-7405-ENG-48.

\section{LAWRENCE LIVERMORE}

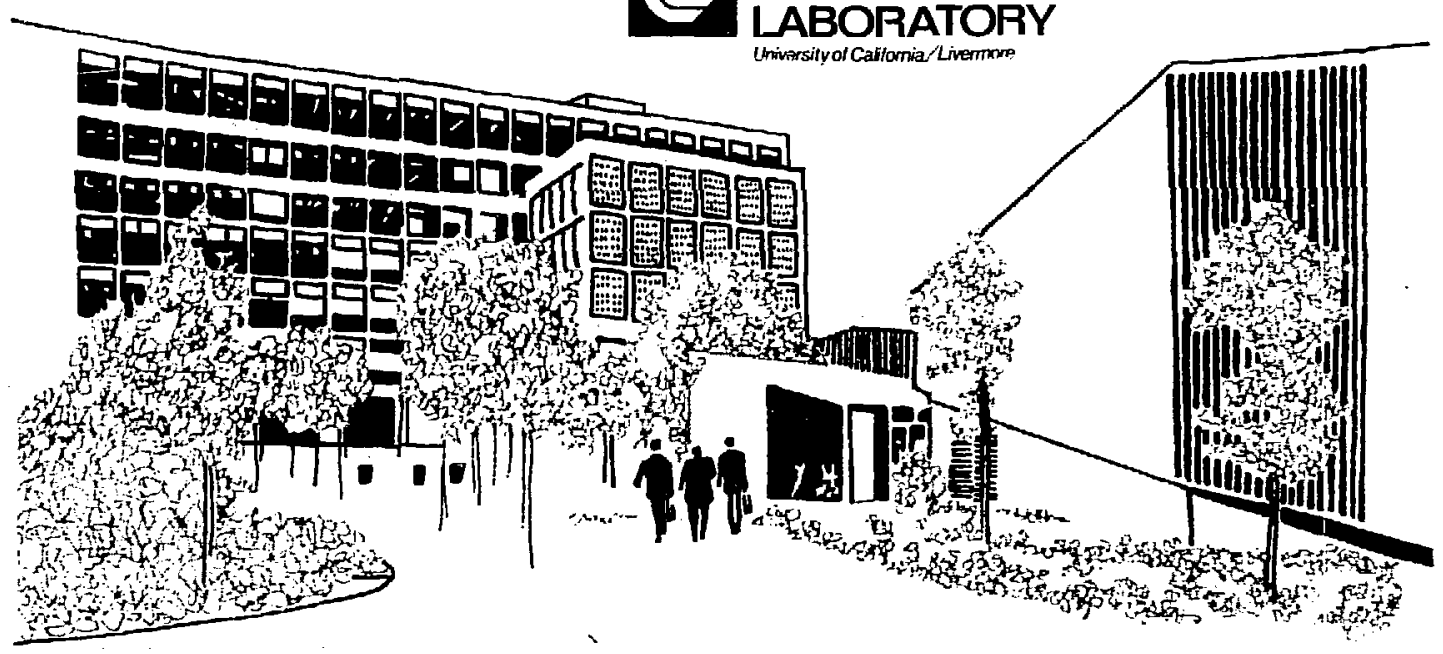




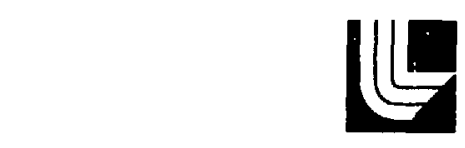

\title{
I_AWRENCE LIVERMORE LABORATORY
}

Universify of Calfornia Livermore,California 94550

UCAL-52806

\section{ENHANCED COUPLING AND DECOUPLING OF UNDERGROUND NUCLEAR EXPLOSIONS}

\author{
R. W. Terhune \\ C. M. Snell \\ 4. C. Rudean
}

Manuscript date: September 4, 1979

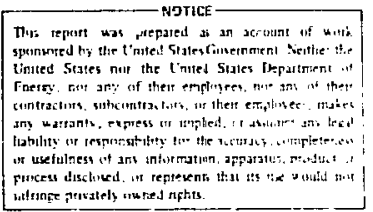




\section{CONTENTS}

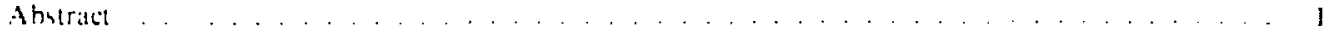

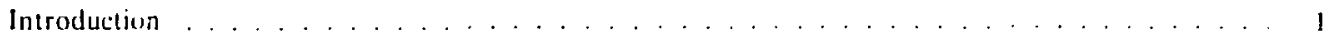

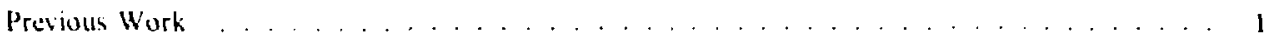

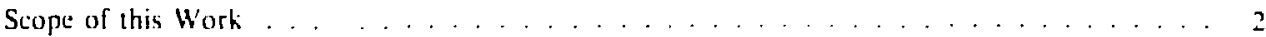

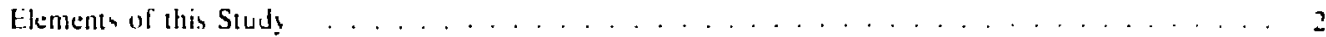

The SOC Code . . . . . . . . . . . . . . . . . . . . . . . . . 2

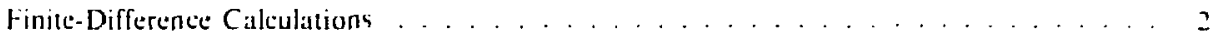

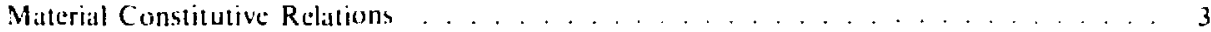

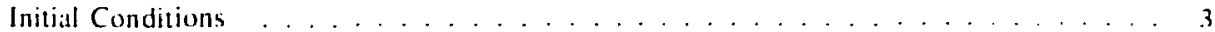

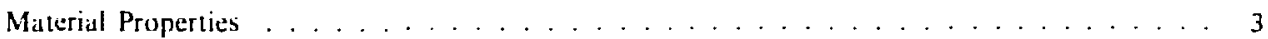

Hardhat Granite . . . . . . . . . . . . . . . . . . . . . . . . . . .

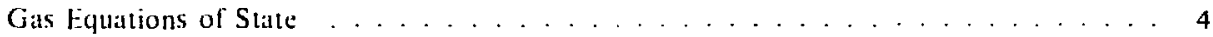

Study Parameters . . . . . . . . . . . . . . . . . . . . . . . . 4 4

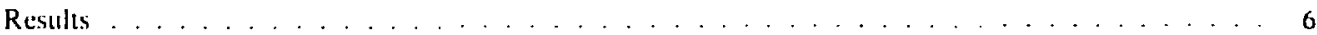

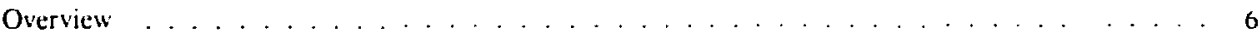

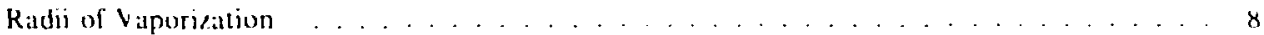

Stress Wave Attenuation . . . . . . . . . . . . . . . . . . . . . . 12

Elastic Response . . . . . . . . . . . . . . . . . . . . . . . 15

Discussion with Respect to some NTS Nucleat Events . . . . . . . . . . . . . . . . . . . 20

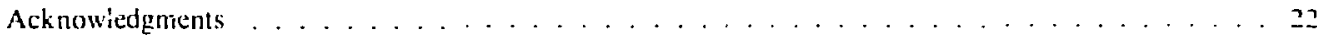

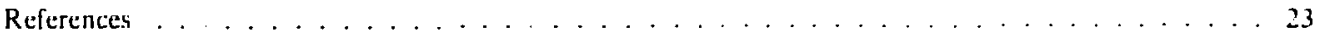

Appendix A-Initial Conditions . . . . . . . . . . . . . . . . . . . . . 24

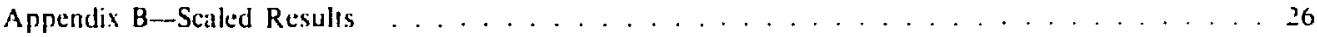




\section{ENHANCED COUPLING AND DECOUPLING OF UNDERGROUND NUCLEAR EXPLOSIONS}

\section{ABSTRACT}

We studied the scismic coupling efficiency of nuclear explosions in granite by means of computer calculations als a function of sealed explosion source radius. The scaled source radii were varied from $0.1 \mathrm{~m}^{\prime} \mathrm{kil}{ }^{3}{ }^{3}$ (point source) $1020 \mathrm{~m} / \mathrm{kt}^{1}$ '(representing a nearly full decoupling cavity). It was foun' that seismic coupling efficiency is at a maximum when the scaled source radius is approximately $2 \mathrm{~m} / \mathrm{kt}^{1}$ ! The primary caluse of this maximum in seismic wave source strength is the effect of initial source radius on peak particle velocit! and pulse duration of the outgoing elastic wave. A secondary cause is that rock vaporizalion (an energy sink; does not occur for scaled source radii somew hat greater than $1 \mathrm{~m} / \mathrm{kl}^{\prime}$ ? Therefore, for scaled source aadii greater than $1 \mathrm{~m} / \mathrm{kl}^{\prime}$, there is additional energy available for seismic wave generations. A wallable data for sone nuclear explosions at the Nevada Test Site do not provide sufficient evidenece to either support or negale the erhanced coupling that is indicated by calculations al scaled sourec radii of $1-2 \mathrm{~m} / \mathrm{kl}^{1}$ ?

\section{INTRODUCTION}

During an AFTAC Suismic Review Pancl meeting on 1-2 June 1976, Trulio of Applied Theory, Inc., reported some interesting results from calculations of the seismic coupling of underground nuclear explosions. He performed it series of calculations for explonions in various sizes of spherical cavities in grantle. The energy gields of the explosions and the masses of the explosive soisces were held constant as the eavity size was varied. Trulio lound that, as cavity volunte was increased from that for a "tamped" explosion to that for a "decoupled" explosion, the strength of the seismic source function first increased somewhat, then decreased substantially at larger calvity volumes. The maximum seisnic signal resulted in a cavily volume somewhat gres:ter than that of the explosive, but much less than that of a full-decoupling cavity. He proposed the following explanation: Seismic coupling reaches maximum efficiency when little or no rock is melted and vaporized, leaving more energy available for mechanical work on the rock. Coupling efficiency declines for source sizes either smaller or larger than this optimum size. Trulio referred to the effect as "enhanced seismic coupling," since an optimum cavity size for seismic energy coupling from al given source is indeated. He suggessed that independent calculations be performed to verify this effect. APRA informally asked linat the Liswrence Livermore Laboratory make such an insestigation. Our independent calculations are discussed in this report.

\section{PREVIOUS WORK}

In comnection with the Cinome. Salmon, and Sterling explosions, Patterson made a series of calculations of tamped. partially decoupled. and fully decoupled explosions in salt and in granite. 1.2 His calculations did not reveal any enhanced coupling at small cavity radii, but they did indicate decreased coupling with increased ratio of cavity volume to explosion energy. This is because Palterson did not make any calculations for the small cavity volunes al which Trulio noted coupling enhancement.

Patterson 's calculations concentraled an near fully decoupled explosions where the scaled cavity or source radius $\left(\mathrm{R}_{0}: \mathrm{W}^{1 / 3}\right)$ is approximalely $30 \mathrm{~m} / \mathrm{kl}^{1}{ }^{3}$ for salt and $20 \mathrm{~m} / \mathrm{kt}^{1}{ }^{3}$ for granite. The 
ealculated normalised reduced displacement poieritial (RDP) for the fully decoupled explosion is on the order of $3.5 \mathrm{~m}^{3} / \mathrm{kt}$. The sciled source radius $\left(R_{1}, W^{\prime}\right)$ for the partially decoupled cialculations exceedel $13 \mathrm{~m} / \mathrm{kl}^{1}$ ?, which is much larger than the range of scaled source radii for which Trulio noted enhanced seisnic coupling.

Patterson compared the effect on the RDP of the detailed explosion phenomena in the cavity with that of assuming the energy is initially distributed uniformly throughout the catvity. For large calvities that would fulty decouple an explosion, the detailed carity pienomena calculations produced the sar.te resulfs it secismic frequencies as calculations based on a miform initial distribution of energy. Howevir, for partially decoupled explosives, the detatled cavity-phenomena calculations gave $\mathrm{R} D \mathrm{P}$ values that ranged from one-tenth to three limes those or the uniformly distributed energy model, depen ling on the mass of the nuclear explosive and the se led souree radius. The larger the mass o! the devict, the greatler the difference hetween the results for the two models.

\section{SCOPE OF THIS WORK}

This report presents a series of numerical (al) :ulations performed with LLL's underground explision computer code SOC. ${ }^{3-6}$ The calculations ar: : intended to simulate detonations of nuclear explosives in various sizes of cavities in granite ap$\mathrm{p}$ oximatcly $1000 \mathrm{~m}$ deep. The primary purpose of t'ic calculations was to determine how the seismic source function (RDP) varies as the initial cavity i.ize is increased from that of an almost point source to that of as fully decoupled tavity. In addition, if Trulio's "enhanced scismic coupling" was obserted in our calculations. ne would allempt to determone the phesseat caluse of that enhancement

The source lerm that we used for the calculaloms is modeled by a sphere of vaporized iron in which the energy and mass of a nuclear device are uniformly distributed throughout the cavity. This results in a uniform pressure throughout the initial cavity, which initiates the propagation of the shock. wave into the surrounding granite medium. This, source model is an excellent approximation of a tamped nuclear detonation, ${ }^{7}$ hut suffers from ofvious defects in simulating the soure function for partially decoupled explosions. We have ignored the detailed cavity phenomenal st udied by Patterson (see above) such as shock-wave propagation through the air between the device and the castity wall, stagna(ion pressure al the cavity wall. impulse of the device dehris impatcting on the cavity wall, and subsequent reverberations caused by shock-wave reflections off the cavity wall. The average cavily pressure during the subsequent cavity expansion, however, is believed to be a reasonable approximation to physical reality. Thus, this parametric study should be viewed as an effects study whore the energy density, mass density, and source size are varied rather than as a definitive study of partially decoupled detonations.

Part of this study is similar to that done hy Trulio in that we used a constant energy source as the eavity size was varied. In addition, we studied the effects of keeping the cavity size constant and varying the energy of the explosion. We rorrelated the results by upplying the principles of similitude as in Hopkinsun Blast Scaling. ${ }^{k}$

\section{ELEMENTS OF THIS STUDY}

\section{THE SOC CODE}

\section{Finite-Difference Calculations}

Calculations discussed in this report were made with SOC, a one-dimensional Lagrangian computer code. ${ }^{3-6}$ Planar, cylindrical, ancl spherical geometries are available; we used spherical geometry in this study. SOC models material motion and stress-wave propagation within a grid of discrete material zones. Motion is initiated by an energy source, stress, or velocity condition introduced within the grid, or by an external force applied to the grid. Given the initial stress field, the code solves the equation of motion to obtain the acceleration at each node within the grid. Acceleraions acting over a small time interval $\Delta t$ produce new velocities. The integrated velocities give displacements, which in turn determine new strains. Finally, these strains are used to calculate new stresses for each zone. A general constitutive relation or stress-strain relation describing the behavior 
of the matcrial is necessary to calculate these new stresses (sec below). Time is then increased by $t$. and the entire process is repeated. The calculation maty be carrled forward in this manner to examine atress-wase propagation throughout the grid.

\section{Material Constitutive Relations}

The physics of malerial behavior is modeled by the eonstitutive relations used $t$ c calculate the tresses an a lunetion of the strains. Thus, the constitutive models are of crucial importance with respect to the physical validity and accuracy of predictive culculations. The results of this study are constrained by the constitutive models used.

The lype of constiturive model used depends in part upon the demands of the problem to be solved. The nuclear-explosion problem involves a broad range of peak stresses, and widely varying regimes of material hehavior. A nuclear explission abrupty releases large amounts of encrgy within the small volume of the explosive assembly. If the event occurs in a tamped configuration (ciosely coupled to the surrounding material), a large stress discontinuity forms and moves outward. This shock wave compresses the material, distributing internal and kinetic energy as it propagates. Close to the source. material is vaporized and acts as a fluid. Peak stresses decrease as a result of spherical divergence of the wavefront and energy deposition in the source vicinity. At greater ranges, a region of melted material is formed, and the water component (if any) may be vaporized. This is surrounded by a region of completely crushed and shaticred material. Fractured or ductilely distorted material extends outward a considerable distance from the source. Finally, gross inelastic effects cease to occur and the wave propagation becomes almost elastic. This series of events occurs at stresses ranging from about $1000 \mathrm{GPa}$ (vaporized rock) down to $0.01 \mathrm{GPa}$ or less (elastic or quasi-elastic wave propagation).

\section{Initial Conditions}

The initial state or starting condition of the computational grid must bc specificd. All of the calculations discussed here use a grid consisting of two concentric spherical regions: a gas material region representing the energy source, and an initially solid region outside the source. The zone size in the source region is constant, while the zone size in the solid material may be either constant or increasing in geometric progression away from the source. The source region may be modeled as an iron gats sphere (representing the explosive atsimbly) or as a sphere of uniform rock gas smulating the eniire volume of rock vaporiecd by the detonation. In this study we used an iron gas source. We assumed that the source region has uniform initial material density, energy density, and pressire. The initial density and total energy are chosen to medel the mass and energy release of the nuclear explosive. The source radius is based on the size of the initial eavity volume for both "iamped" and "decoupled" explosions.

An initial stress distribution mist also be specified for the rock mediun outside the cavits. The werburden stress plays a role in halting cavity expansion for decely buried events: it is particularly important in the case of decoupled explorions beciuse the final carity pressure is approximately equal to the werhurden pressure. Analytic solutions are: available for certain cases of interest, such as a spherical cavity in an externally pressurized linear elastic medium. No analytic solutien exists for the more general casc of a spherical cavity in a gravitationally settling mediun. However. numerical techniques may be used to solve this problem. One convenient method developed by Hancock ${ }^{9}$ uses a finite-difference seheme to perform mechanical-equilibrium calculations. The method consists of adding a velocity-damping term to the equation of motion in a finite-difference calculation. The equation of motion is then integrated stepby-step with respect to a time-like variable, called "pseucu-time." Proper thoice of the damping constant allows rapid convergence to mechanical equilibrium. This mechanical-equilibrium solution capatility was added to the SOC code. and was used to establish initial conditions for some of the decoupling calculations.

\section{MATERIAL PROPERTIES}

\section{Hardhat Granite}

Material properties needed for the calculations include the initial density and elastic constants of the medium, energy content required to liquefy and viporize, vapor equation of state. ${ }^{10}$ and the compressibility and shear strength of solid material. This information is normally obtained from laboratory and field measurements, theoretical and empirical models of rock behavior. studies of 
previous tests in similar media, etc. Detaled investigations of the Hardhat and Piledriver events have resulted in the development of a corstitutive description intended to model dynamic effects of laerge-seale nuclear detunations in it dense granitic medium. 11-14 This previously derived description is itdopled for the corrent study. The granite is assumed to be a low-porosity, weak, fully saturated material. The low strength and wet condition are meiant to charaterize the bulk properties of at jointed and imperfeet rock mitss. Small uncracked laboratory specinens show higher strengths. Since amplete siluration is assumed, the material suffers no irresersible compation (atir boid remosal) upon ioading.

The physical properties adopted for the granite medium are listed in Table 1. These paraneters are generally represintative of a dense. Wet gransic formattion, frite values of sume of these parameters are comp: ted in Table 2 with those used b! Trulio.

\section{Gas Equations of State}

The encrgy source region and surrounding vaporized rock play a central role in establishing and maintianing the stress field of the explosion. Thus. it is important to hate an adequitc deseription of the gas materials. Theoretical models describe the hehavior of atomic, molecular, and ionic mixtures within certain regions of energy and density. These models have been incorporated into computer codes which cialculate equilibrium statc points within the appropriate regions. Butkovich has used the theoretical equation-ol-state codes. in conjunction with experimental data, to estiblish gas equations of state for at vatrity uf natural mallerials. ${ }^{10}$ The results are plotted ats pressure vs internal energy for various isochores or curves of constant density. The calculated equations of state include densities from $10^{-5}$ to $10 \mathrm{Mg} / \mathrm{m}^{3}$, an interval which encompasses the region of interest for most explosion calculations. The SOC code accepts corresponding tabular equations of state that give pressure as a function of internal energy and density. A two-dimensional interpolation procedure is used to calculate points that lie between two isochoric curves. We used equations of state for iron gas and for a mixture of $\mathrm{SiO}_{2}$ plus $\mathrm{I} \%$ by weight of water vapor to represent the rock.

Most geological formations contain significant amounts of naturally occurring water. Thus, water vaporization effects may influence dynamic interac- tions close wo the detonation. Since valer vapories at much laner strese ferels than does the associaled rock malerall, a neans must be provided lo take inlo account the partial-pressure contrshution of water vaper. The SOC code utilies at technique developed hy Butkovich. ${ }^{15}$

\section{STUDY PARAMFTERS}

We made three series of explosion calculations: 11 in the "taneped" (T) series, 15 in the "decoupled" (D) series, and 7 in the "decoupled and relaxed". (DR) series. which are sunmarized in the lables in Appendices $A$ and $B$.

In the $T$ series, yield (W) was varied from I to $1(0) 0 \mathrm{kt}$. the initial cavity radius $\left(\mathrm{R}_{(1)}\right)$ was viried from $0.101 .20 \mathrm{~m}$, and the initial iron gits density $\left(m_{1}\right)$ was varied from $1.5103 .23 \mathrm{Mg} / \mathrm{m}^{3}$.

In the D series. $W$ was constant at $1 \mathrm{kt}$ except for (wo calculations with $W=1000 \mathrm{kt}$. where $\mathrm{R}_{0}$ was varied from $1.2 \mathrm{w} 15 \mathrm{~m}$. and $p_{0}$ wals varied from $4.18 \times 10^{-1} 100.817 \mathrm{Mg} / \mathrm{m}^{3}$.

In the DR series, $W^{\prime}$ was $1 \mathrm{kt}$ in all cases: $\mathbf{R}_{0}$ "als varied from 2.5 to $23.62 \mathrm{~m}$. and $m_{0}$ was varied from $2.47 \times 10^{-5} 109.04 \times 10^{-2} \mathrm{Mg} / \mathrm{m}^{?}$.

The principal difference between the $D$ and DR series wils in the initial rock steess distribution surrutunding the catvity. A uniform hydrostatic pressure throughout the granite of $26 \mathrm{MPa}$ was assumed in hoth the T and D series. In the DR series. the preexplusion stresses in the rock surrounding the cavity were relaxed to an equilibrium state corresponding to rero cavity pressure and an overhurden pressure of 26 MPa. except in DR6 and DR7 where an werhurden pressure of 18.1 MPa was used.

Our reference "tamped" explosive was assumed to have a mass of $5.91 \mathrm{Mg}$. an equivalent spherical radius of $0.98 \mathrm{~m}$. and a corresponding density of $1.5 \mathrm{Mg} / \mathrm{m}^{3}$. Unlike conventional explosives, the energy release from nuclear explosives is almost independent of the mass and volume of the explosive assembly. Two nuclear explosives can have approximately the same mass and volune but have yields that differ by an order of magnitude or more. Both explosives could be emplaced in a "lamped" configuration, but would have different seismic source characteristics. Similarly, both explosives could be emplaced in a "decoupling" cavity, but their seismic soure characteristics nould differ. Therefore. we summarized our results 
TABLE I. Material properties for Hardhat granite.

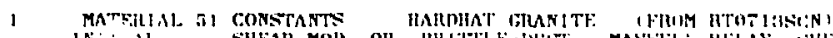

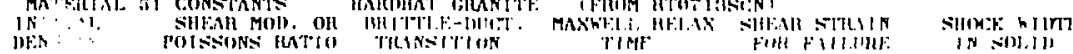

a.

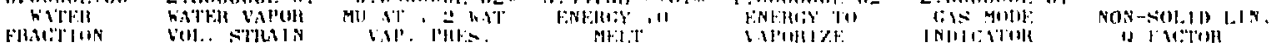

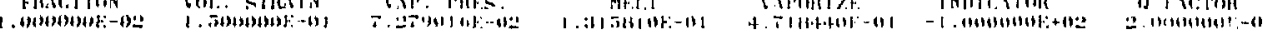

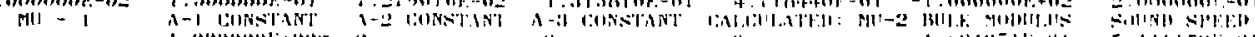

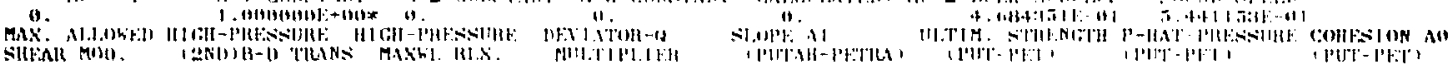

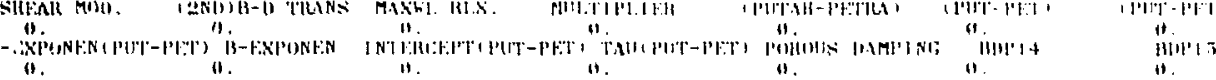

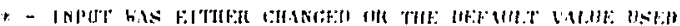

\begin{tabular}{|c|c|}
\hline & IMNBIN: Th \\
\hline & \\
\hline 1. nowout:-61s & a. $-62(1+5-116$ \\
\hline $2.016(36)(6)-6): 3$ & :3.729475-61 \\
\hline $3.0(3)(1)(+1)-(1:)$ & 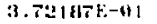 \\
\hline 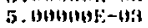 & $3.7052(1)-(1)$ \\
\hline 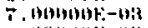 & $3.019345 k_{-}-111$ \\
\hline $1,(1+1+1,(1)+-102$ & :3.67336t:-61 \\
\hline $1,50+1+0+35:-612$ & a. $2472\{1,-61$ \\
\hline 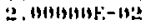 & $3.62+7220-61$ \\
\hline 3. & 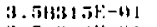 \\
\hline 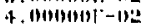 & $3.542(11) t-611$ \\
\hline 6. $61616(+6):-162$ & $: 1.4513(+1)+-1+1$ \\
\hline 1 . 10606obt:-11! & $3, \geq 6,(1) 1):-11$ \\
\hline 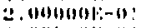 & 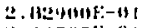 \\
\hline 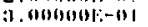 & $2 .+4$ nons:- -131 \\
\hline +. & $2.252011+10 t-111$ \\
\hline 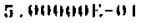 & 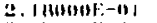 \\
\hline $8,4161664+1:-41$ & ב,, 7 (a) \\
\hline I. (1)16(1) $15+111$ & 1. $4050601-01$ \\
\hline 1. $-7(1)(1+1)+(16)$ & 1. y/:ulur.-1)i \\
\hline & 1. Bothathl:-ol I \\
\hline $64 f+411$ & 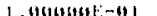 \\
\hline
\end{tabular}

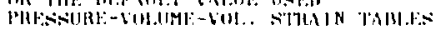

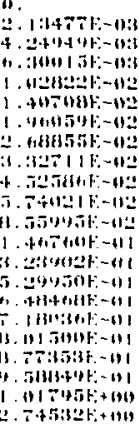

\begin{abstract}
III) $\|$ IIII
\end{abstract} . pistitis:-0

4. $6(3)+355-61$

4. $423-5 \mathrm{E}-111$

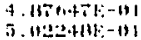

5. $142.3+115-191$

$5.4+4+5 k-11$

T. diwi:-

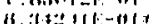

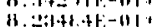

(1)

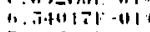

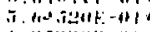

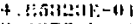

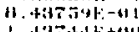

$1 .+33+2++4+111$

$2.3962:+5+161$

3. $1: 5,3+4:+3111$

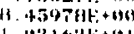

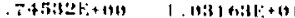
"

1

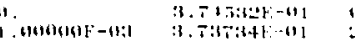
(16)16(1)- ll:

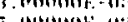

3.

$(3+1+1)+11:-112$

$50+1+1+5:-60$

a.monst:-1t:

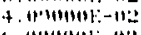

1. . 1616 (1)

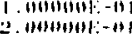

1. fontmat-111

4. monmol: -

.

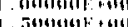

a cuscount

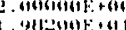

1. Ah:ente th

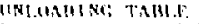

\section{(1) $7: 3: 1+1+11$}

3.

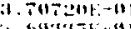

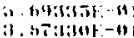

a. 64t2at -

a.

3. ה.

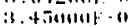

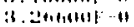

2. Hasest-o

ב.t.thumb! on

2-20111 11

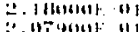

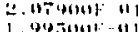

1. 1950116-41

1.

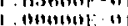

‥ 1:14ร-1:-413

4. $2+41+4610-1366$

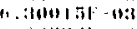

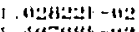

1. 4 (15)

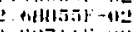

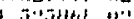

1. 7.

!. 40.5t111: 11

1.

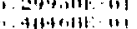

2. |l|blitat on

14.

1. $117+45+1111$ ititit it.

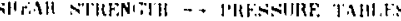

I.AMIII); TABL.

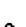

5. $13(1)(1)+6) \mathrm{F}_{-}-(1) 4$

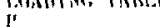
$2.06 \cos (1):-65$

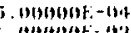

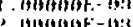

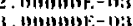

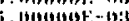

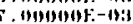
(19)

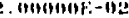
1. (11!)(3)(6):-1):2

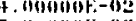

1. menentotion

\begin{tabular}{|c|c|}
\hline \multirow[t]{2}{*}{ IIF: $\mathrm{ml}^{\prime}$} & $\begin{array}{l}\text { PlBAR } \\
\end{array}$ \\
\hline & 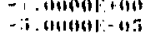 \\
\hline $3|r-21|$ & 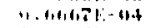 \\
\hline & 1. 20 ent:-nas \\
\hline$(1 F=+4)$ & 2.:B \\
\hline & 1. + +16, 6 - $-6: 3$ \\
\hline WInt:-61 & ; . 1.5601 - $6: 3$ \\
\hline T-3(n)F:-61 & 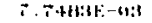 \\
\hline onten:-11 & 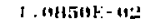 \\
\hline 15131) & 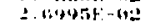 \\
\hline ב-2 & 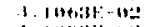 \\
\hline (1606):-1*3 & 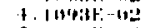 \\
\hline 610:- - 16! & i. $t 10 t 1+-1+2$ \\
\hline & 1. $1913 \mid 111,+1+11$ \\
\hline
\end{tabular}

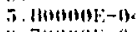

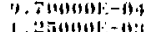

1.

1.

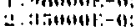

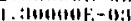

i. . 361616)

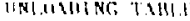

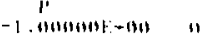

1. A16sust:-10:2

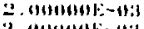

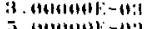

‥(111611)1:-1):

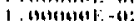

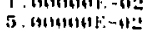

1. (16)61616)+a11
1.1. IP

3.

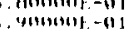

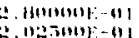
2. $1251195-11$ 1. 20 : ‥
Iif IMII

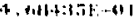

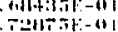

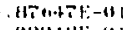
T. $1922+4+15-61$ 5. 4 inestiMel15-20:-101

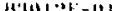
a. :14:011:-110 - A11)

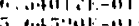
4 4. 11. +15 $514-61$ 1. $4.65+4+41+611$ a M Nint truats

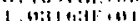

.

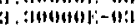


TABLE 2. Material properties assumed for hardhat granite (RT0713SCN) compared with those used by Trulio.

\begin{tabular}{|c|c|c|}
\hline Property & This study & $\begin{array}{l}\text { Truljo } \\
\text { study }\end{array}$ \\
\hline $\begin{array}{l}\text { Initial density at zero pressure } \\
\left(\rho_{0}\right), \mathrm{g} / \mathrm{cm}^{3}\end{array}$ & 2.67 & 2.7 \\
\hline Elastic Poisson's ratio (v) & 0.28 & 0.28 \\
\hline Tulal porosity $(\downarrow)$ & 0.0267 & 0 \\
\hline Saturation (S) & 1.0 or $100 \%$ & 0 \\
\hline Grain density $\left(\rho_{\mathrm{g}}\right), \mathrm{g} i \mathrm{~cm}^{3}$ & 2.7158 & 2.7 \\
\hline Water content by weight $(Z)$ & (1.01 & 0 \\
\hline $\begin{array}{l}\text { Peak pressure to vaporize } \\
\text { water, GiPa }\end{array}$ & I0.18 & - \\
\hline $\begin{array}{l}\text { Encrgy to liquefy granite. } \\
\mathrm{Mb}-\mathrm{cm}^{3} / \mathrm{g}\end{array}$ & 0.0493 & 0.035 \\
\hline $\begin{array}{l}\text { Enctgy to vaporize granite, } \\
\mathrm{Mb}-\mathrm{cm}^{3} / \mathrm{g}\end{array}$ & 0.1767 & 0.035 \\
\hline $\begin{array}{l}\text { Initial zeri-pressure hulk } \\
\text { modulus. GPa }\end{array}$ & 46.84 & 57.6 \\
\hline $\begin{array}{l}\text { Initial zero-pressure somnd } \\
\text { speed. } \mathrm{cm} / \mu \text { s }\end{array}$ & 0.544 & 0.6 \\
\hline Intact tensile strength, $\mathrm{Gl}_{\mathrm{a}}$ & 0.005 & $?$ \\
\hline Intact unconfined strength, Gl's & 0.01 & $?$ \\
\hline
\end{tabular}

in terms of parameters sealed to the energy release with length and time referenced to the cube root of the explosion yield. Our assumed uniform overburden pressure conditions and aro gravily condition are compatible with such scaling. As shown below, there are no clear-cut distinctions betwen "tamped" and "decoupleu" nuciear expiusiuns. Rather, tlese are variations within and a transition between these two classes of explosions, which are a function of scaled source radius.

In Fig. 1, we show the initial iron gas or cavity pressure as a function of scaled initial cavity radius for all our calculations. The initial cavity press:'res

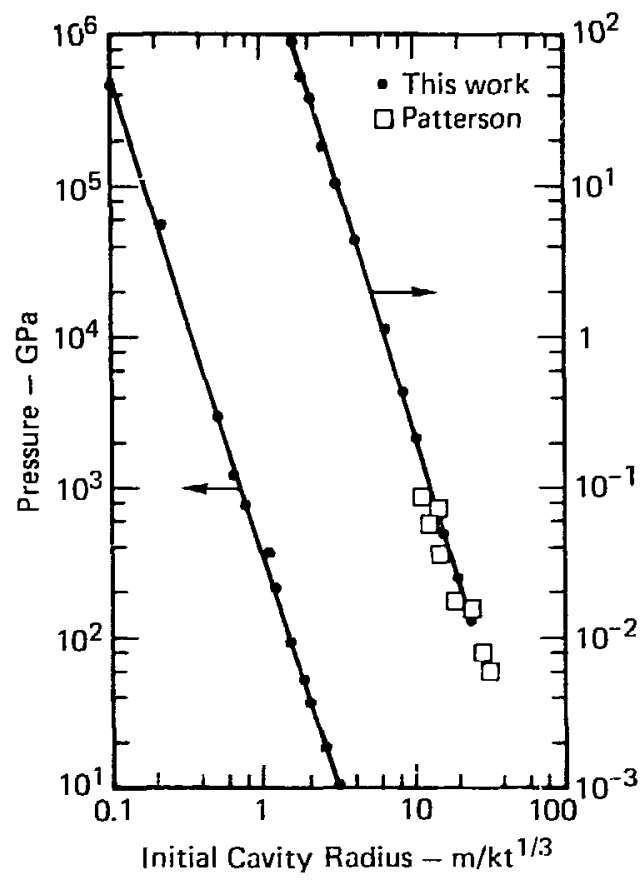

FIG. I. Initial cavity pressure vs scaled cavity radius.

for Patterson's 1.2 calculations are also given. It is shown that Patterson considered cases that are near or at the fully decoupled condition (scaled calvity radii of approximately $10 \mathrm{to} 30 \mathrm{ml} / \mathrm{kt}^{\mathrm{l}}$ 3). By full decoupling, we mean that the explosion does not caluse any inelastic bebavior of the rock. In this work, we have extended the work of Patterson to a near-point source (scaled cavity radius of $\left.0.1 \mathrm{ml} / \mathrm{kt}^{1 / 3}\right)$.

Appendix A gives the initial conditions for each calculation.

\section{RESULTS}

\section{OVERVIEW}

Appendix B gives a summary of the scaled results from each calculation of the parameter study.

The effect, as reported by Trulio, of enhanced seismic coupling for partially decoupled sources was confirmed in this parametric study. Figure 2 is a plot of the final reduced displacement potential (RDP) in the eli: $t$ ic region as a function of the initial cavity radius caled to the cube root of the energy of the device within the cavity. The initial scaled source radii are an inverse furction of the initial encrgy density and vary from $0.1 \mathrm{~m} / \mathrm{kt}^{1}{ }^{3} 10$ 


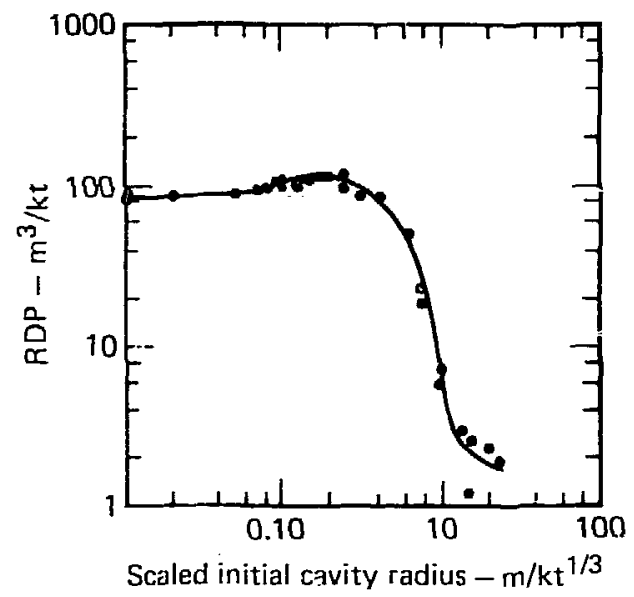

FIC. 2. Final RDP vs scaled initial source size.

about $20 \mathrm{~m} / \mathrm{kl}^{1 / 3}$, which represents a variation between almost a poin: source tamped to an almost fully decoupled cavity. The calculation with an initial scaled source radius of $1.8 \mathrm{~m} / \mathrm{kt}^{1 / 3}$ gave the maximum RDP, which was $47 \%$ greater than the RDP obtained for a near-point source. The RDP for an almost fully decoupled cavity is about 2 to $3 \%$ of that obtained for a point source and is in excellent agreement with the results of Parierson.

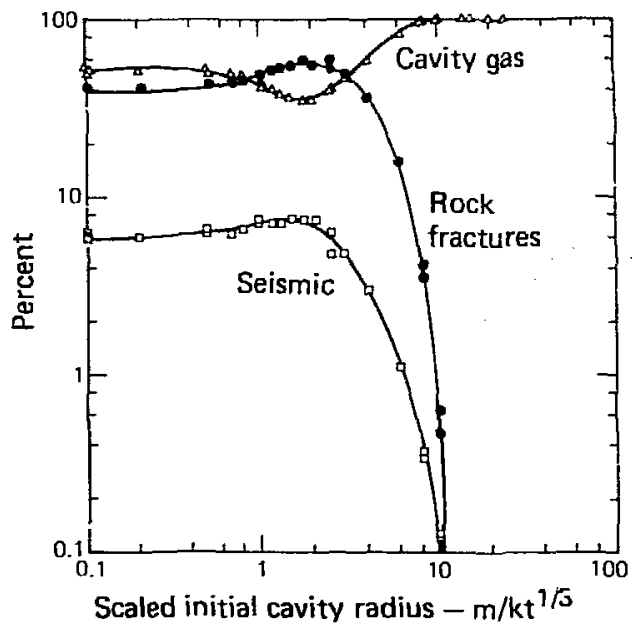

FIG. 3. Final energy balance vs scaled initial source size.
The same characteristics of the RDP curve in Tig. 2 are evident in the seismic energy balance curve shown in Fig. 3. Figure 3 is a plot of the energy halance in three well-defined regions at the id of the calculations as a function of the scaled source radii. These regirns are the internal energy in the cilvity region, the internal energy dissipated in the iructured rock, and the internal plus kinetic energy in the clastic region, which has been designated the seismic energy. At a scaled source radius in the ncighborhood of $2.0 \mathrm{~m} / \mathrm{kt}^{\mathrm{l}} 3$, both the energy trapped in the fractured rock and the seismic energy are near maximum, while the residual internal energy in the cavity is at a minimum. The energy in the cavity includes the potential energy of cavity gas due to its pressure. the energy required to vaporize and melt the rock, and the energy required to vaporize the in situ water. Vaporization of the rock occurs only for scaled source radii less than $1.1 \mathrm{~m} / \mathrm{kt}^{1,3}$, with a significant reduction of the mass of rock viporized compared to that for a point source for scaled source radii between 0.7 to $1.1 \mathrm{~m} / \mathrm{kt}^{1 / 3}$. Nelting of the rock occurs only for scaled source radii less than $2.0 \mathrm{~m} / \mathrm{kt}^{1 / 3}$ and water vaporization occurs for scaled source radii up to $3.0 \mathrm{~m} / \mathrm{kt}^{\mathrm{l}}$. Thus the internal energy of the cavity reflects these various energy-loss mechanisms as the scaled source size is varied from a point source to larger radii. The coincidence of the scaled source size for which melting ceases and the maximum at which the RDP oceurs, suggested to Trulio that the enchanced coupling is due entirely to the additional energy that is not being utilized to vaporize and melt the rock. The paramet-ic study reported here shows that another phenomenon in addition to the energy disposition is involved in the enhanced coupling effect. This will be discussed later.

Figure 4 sliows the scaled radii at which some of the major changes in the state of the rock occurred as a function of scaled source radii. The curves shown in Fig. 4 are the radius of rock vaporization, the radius of in sint water vaporization, the final cal rity radius. and the elas:ic radius or the limiting distance to where fracture occurred. The straight line at $45^{\circ}$ represents the initial source size. The amount of rock vaporized is essentially constant for scaled source radii from $0.1 \mathrm{~m} / \mathrm{kt}^{\mathrm{l}} / \mathrm{i}^{3}$ (point source) to $0.8 \mathrm{mi}^{\prime} \mathrm{kt}^{13}$ and is much larger than the amount of iron gas over this range. Beyond a source ladius of $0.8 \mathrm{~m} / \mathrm{kt}^{1 / 3}$ the amount of rock vaporized decreases rapidly and the iron gas becoms sthe dominant cavity gas. 


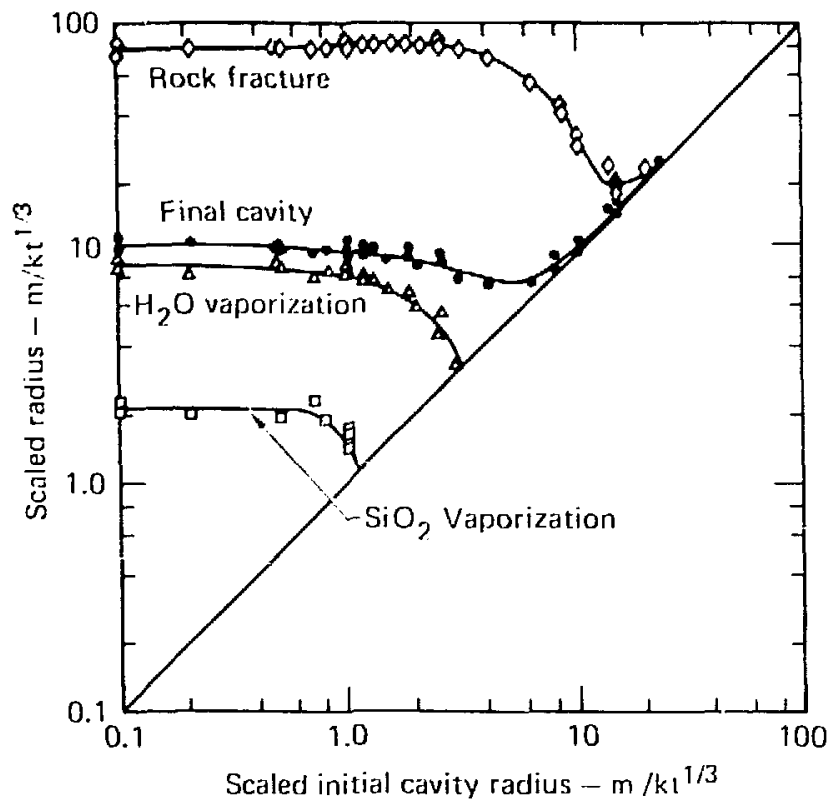

Flli, 4. Kadii of major states of rock vs scaled initial source size.

The sealed distance to which the in situ water is vaporized is essentially constant for seitled source ratdii between $0.1 \mathrm{to} 1.0 \mathrm{~m} / \mathrm{kt}^{1}$. theil decrealses 10 zero alt a sealed source radius of $3.0 \mathrm{~m} / \mathrm{kl}^{13}$. The offect of the water vaporization wats deemed negligible on the enhanced seismic coupling.

The elastic radius is shown to be essentially constant over the range of sealed source radii between 0.1 and $3.0 \mathrm{~m} / \mathrm{kt}^{1 / 3}$. The elastic radius varies between $80-85 \mathrm{~m} / \mathrm{kt}^{1 / 3}$ with a broad peak al about a scaled source size of $2.0 \mathrm{~m} / \mathrm{kt}^{1 / 3}$, in agreement with the RDP curve (Fig. 2). Beyond a sealed source radius of $10 \mathrm{~m} / \mathrm{kt}^{1 / 3}$. very little of the rock is fractured and the stress wave is essentially elastic beyond the source cavity boundary.

Figure 5 shows a comparison of the final cavity pressure with the initial cavity pressure as a function of scaled cavity radii. As the initial source cavity is increased to larger values from a point source, the final cavity pressure also increases until a peak is reached at $R_{0}=6.0 \mathrm{~m} / \mathrm{kt}^{1 / 3}$. These high cavity pressures have interesting implications for the containment of the radioactive gas for partially decoupled events. 16

\section{RADII OF VAPORIZATION}

The ploss af reduced displacemem polentiat and other physical yuantities shou an ..pparent discontinuity near a scialed source radius it $0.98 \mathrm{~m}^{\prime} \mathrm{kt}^{13}$. The RDP. for example. increanes b! about $11 \%$ belween source radii of 0.686 and $0.98 \mathrm{~m} / \mathrm{kl}^{1 / 3}$ (Fig. 2). It then increatses more slouly and continuously alt radii just above $0.98 \mathrm{~m} \mathrm{kl}^{1}$ ? This at rupt change in behavior may be explained in terms of rock vaporization by the nuclear event. Those detonations with initial source radii less than about $0.98 \mathrm{~m} / \mathrm{kt}^{1 / 3}$ vaporize very large masses of surrounding rock. Events with inilial raddii nealr $0.98 \mathrm{~m} / \mathrm{kt}^{1 / 3}$ fully vaporize a significantly smaller amount of rock, corresponding to only a few zones in the finite-difference grid. No vaporization occurs for source radii greater than 1.1 to $1.2 \mathrm{~m} / \mathrm{kl}^{1}{ }^{3}$ in these calculations; of course. some heating, liquefaction, and water vaporization may still take place for larger-radii sources. This Iransition from vaporizing to non-vaporizing behavior occurs rather abruptly because the initial pressure and energy density drop off rapidly with increasing 


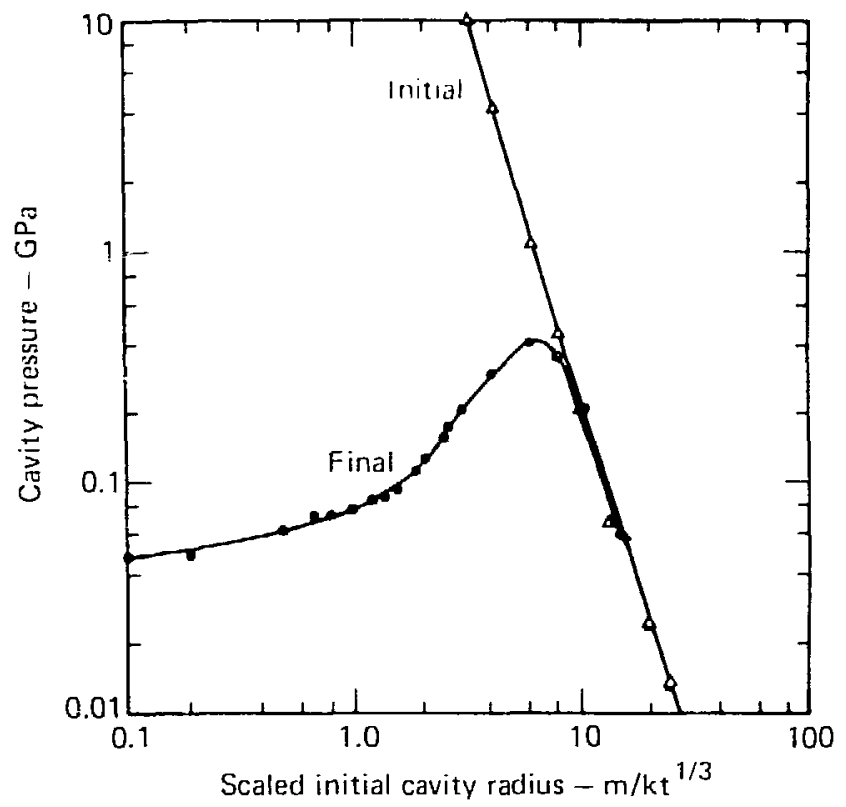

FIC. 5. Initial and final cavity pressure is scaled initial source size.

source radii. The transition radius at which rock vaporization ceases to play a crucial role lies very close to $0.98 \mathrm{~m} / \mathrm{kt}^{1}$.

In order to investigate the relationship between rock vaporization and energy coupling. we have closely examined three typieal I kt calculations, i.e., events $T 6, T 9$, and $T 10$, for source radii $R_{0}$ of $0.686 \mathrm{~m}, 0.98 \mathrm{~m}$, and $1.2 \mathrm{~m}$. The corresponding initial source pressures were $106 \mathrm{GPa}$ (extensive rock vaporization), $38 \mathrm{GPa}$ (small amount of rock vaporization), and $20 \mathrm{GPa}$ (no rock vaporization). The initial source aensities were $1.5 \mathrm{~g} / \mathrm{em}^{3}$ in all cases. The zoning varied somewhat among the three problems, but results were similar for other comparable calculations and were not critically influenced by zone size. Figure 6 shows the iron gas source mass, the mass of rock vaporized, and the mass of material with the water component vaporized for the three events. Note that the mass of rock vaporized is large for the $0.686 \mathrm{~m}$ event, but is much smaller and hardly greater than the iron gas source mass for the $0.98 \mathrm{~m}$ event. No rock was vaporized in the $1.2 \mathrm{~m}$ event.

The influence of energy-source pressure and shock-loading behavior of the surrounding rock on energy coupling has previously been studied. ${ }^{17}$ The peak pressure and the response of material to the outward-going shock wave govern the amount of encrey los! to shock heating and thereby the closerange coupling efficiency. Figure 7 schematically shows the response of a dense solid material of initial specific volume $V_{0}$ to shocks of varying amplitude. For low-pressure loading. the material loads along a Rayleigh line and unloads close to the initial Hugoniot line (little shock-heating effect). The PdV expansion work available from the material is approximated by the area under the Hugonior line, while the waste heat or energy loss that does no work on the surroundings is given by the area between the Rayleigh line and the Hugoniot line. The waste heill is seen to be relatively small in this case. For high-pressure loading. the material loads along a much steeper Rayleigh line. The increased internal energy is sufficient to cause vaporization. The hot vapor material unloads to expanded (larger) specific volumes at low pressures. In spite of this increased expansion, an enormous amount of energy is lost as excess waste heat. Thus, much of the energy is retained as internal heat and energy coupling to greater ranges is actually less efficient for the highpressure loading case (vaporized material). Those 


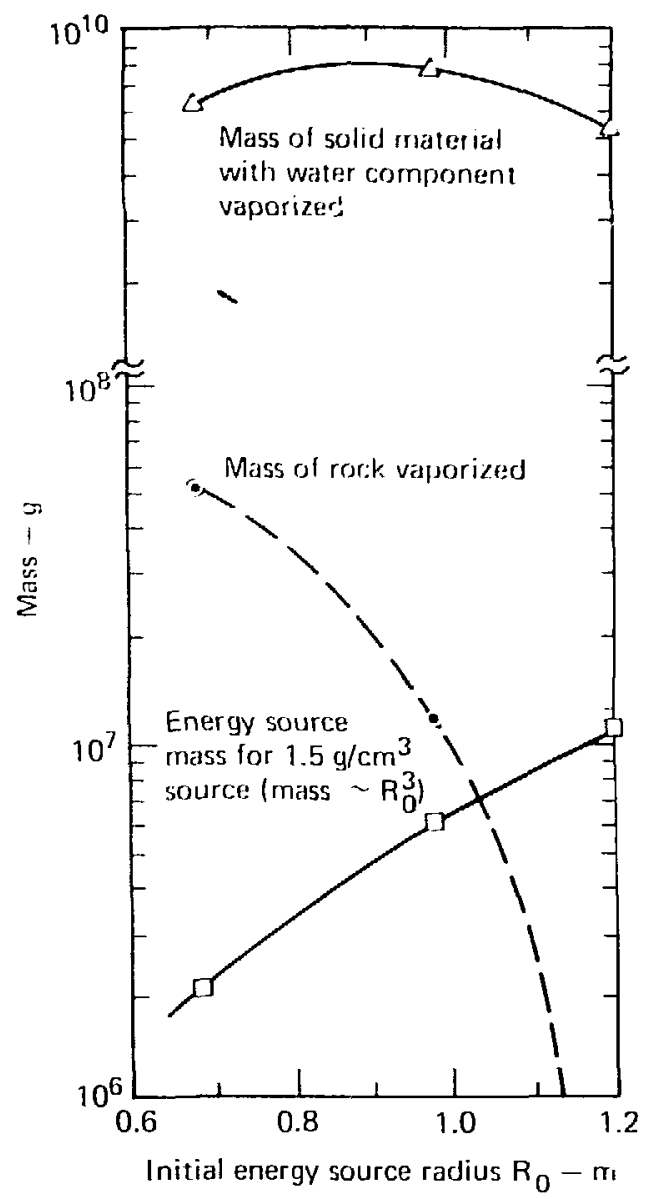

FIG. 6. Vapor material masses for $1 \mathrm{kt} \mathrm{SOC}$ calculations, source radii $k_{0}=0.686$ to $1.2 \mathrm{~m} / \mathrm{kt}^{1 / 3}$.

events that vaporize a significant amount of rock $\left(\mathrm{R}_{0}<0.98 \mathrm{~m} / \mathrm{kt}^{1 / 3}\right)$ will suffer large shock-heating energy losses of this type, resulting in less-efficient coupling.

The approximate late-time volumetric expansion paths for the iron gas sources in the three sample problems are shown in Fig. 8. The source regions unload to a pressure of about $0.1 \mathrm{GPa}$ at a time of $10 \mathrm{~ms}$, the cutoff time for this plot. The small-radius problem $R_{0}=0.686 \mathrm{~m}$ has the highest expansion path and the greatest final volume expansion, because it started at the highest initial pressure and internal energy density. However, the final iron gas radius $\mathbf{R}=7.107 \mathrm{~m}$ is smallest in this case because of the smaller initial radius. The expanding

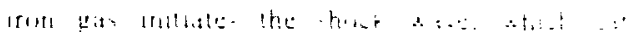

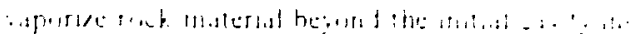

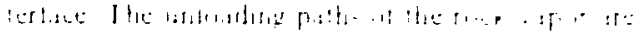

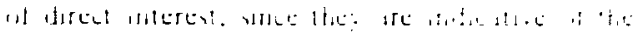

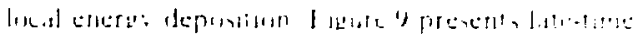

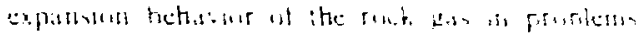

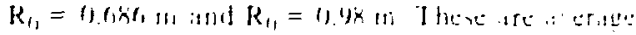

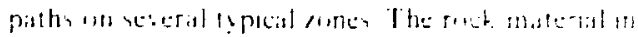

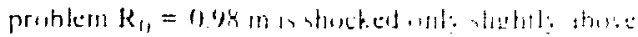

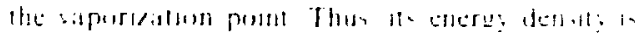

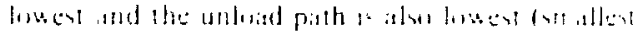

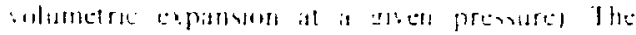
:apur ante tarthes from the undere at prothem

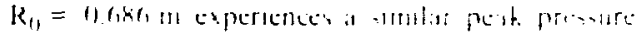

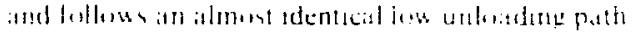

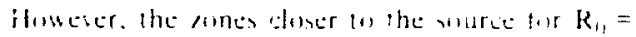

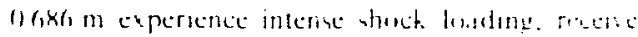

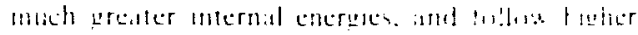

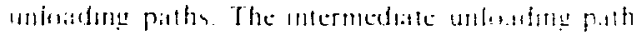

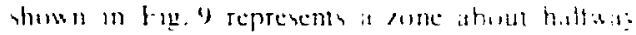

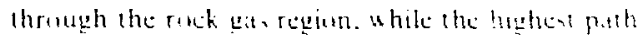

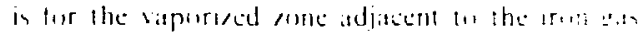
milset

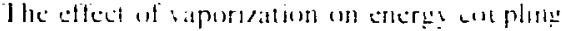

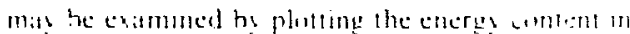

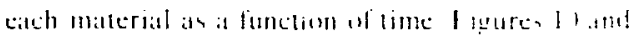

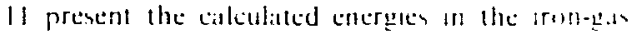
source region, rock-vapor reggon. saportad-atice regull, and sulid granile region as funclumsul tume These energies are divided inlo hinetic and merna!

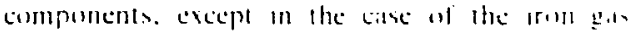

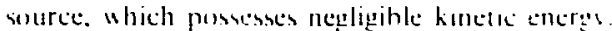

Figure io demonstrales that the cheres retained in the iron gats decliness stcadils with ume. as the source expands and couples to the ensironment. The relative amount al eneres in the wurce at a given line is greatest for the $1.2 \mathrm{~m}$ source (latgest. most massive source regionl. and is least for the smiall $0.686 \mathrm{~m}$ source.

The energy contained in rock vapor presents : different picture (Fig. I0). Both the internal and kintetic energies are greatest for the $0.686 \mathrm{~m} \mathrm{enent.}$ and decrease by a factor of four to six for the $0.98 \mathrm{~m}$ event (which vaporized only a small amount of rock). The $1.2 \mathrm{~m}$ event did not vaporik an! rock material. Note that the internal energy in rock vapor is a very significant fraction of the wal energy fin the $0.686 \mathrm{~m}$ source. This filct is closel! related to the waste-beat energy loss and the lessefficient coupling for the 0.686 in event. Energ! deposition in rock vapor is snall or nonexisten! for the other two cases. 


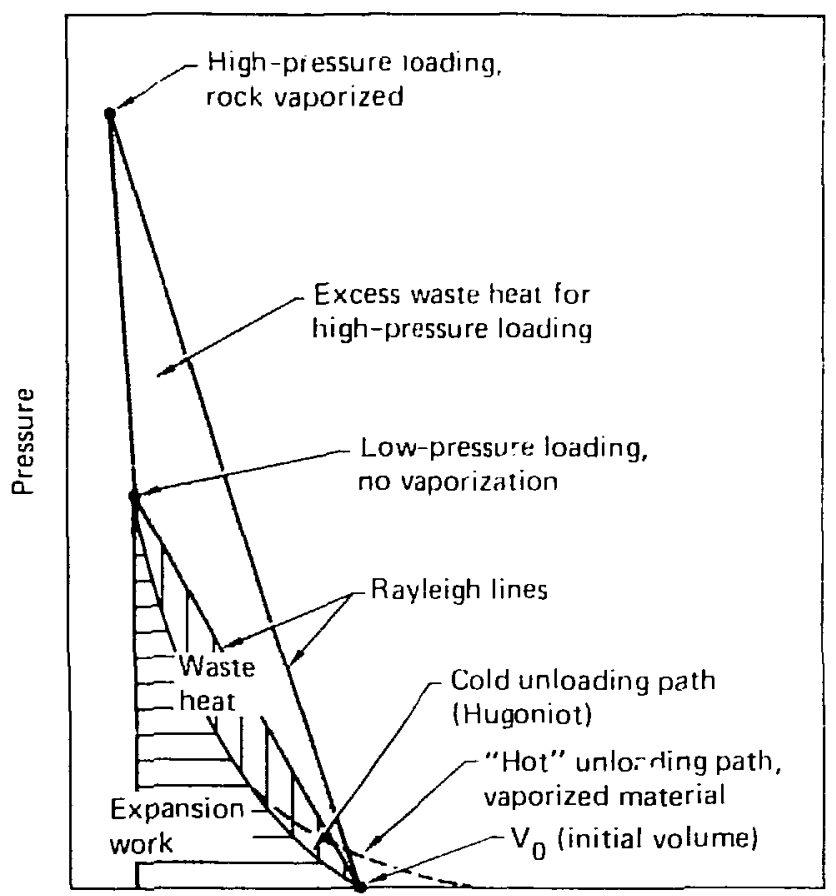

Specific volume

FIC. 7. Shock loading and vaporization of rock. ${ }^{17}$

The internal energy in the oaporized water region shows a similar trend (Fig. 10): it is greatest fur the $0.686 \mathrm{~m}$ extent (strong shock at close ranges), slightly less for the $0.98 \mathrm{~m}$ event, and much !.:ss for the $1.2 \mathrm{~m}$ event. The kinetic energy in the vapurizedwater region displays somewhat less consistent behavior, with the highest value (at times after $1 \mathrm{~ms}$ ) for the $0.98 \mathrm{~m}$ event, a lower value for the $1.2 \mathrm{~m}$ event, and the lowest value for the $0.686 \mathrm{~m}$ event. This difference is due in part to the lower coupling efficiency for the $0.686 \mathrm{~m}$ event, which tends to decrease kinetic energies coupled everywhere outside the rock-vapor region. The difference is also partly attributable to the mass of material in the vaporized-water region, which is greatest for the $0.98 \mathrm{~m}$ event and slightly less for the other two events (Fig. 6).

Finally, the kinetic and internal energies in solid rock are presented in Fig. 11 . These energies have not been plotted separately for fractured and unfractured material, since rock failure is still occurring at $14 \mathrm{~ms}$ and the wave propagation will not hecome elastic until much later in the exent histors. The internal-energ! curves croso at timen earlier than $5 \mathrm{~ms}$ hecause of close-range coupling effects. However, after this time. the kinelic and internal encrgies in rock are alwags greatest for the $1.2 \mathrm{~m}$ event. less for the $0.98 \mathrm{~m}$ event, and least for the $0.686 \mathrm{~m}$ event. This outcome is completely consistent with the calculated displacements and reduced displacement potentials at very late times, which also show the most efficient overall coupling for the $1.2 \mathrm{~m}$ event and least-efficient coupling for the $0.686 \mathrm{~m}$ event. These energy results strongly support the proposed source-cou, "ing explanation, i.e.. the small-radius source generate very high pressure loading, vaporization. and waste-he. $t$ losses at close range. Thus, it couples less efficiently with the surrounding medium than a somewhat larger effective source of lower initial pressure.

It is of interest to closely examine kineticencrgy transfer to the rock for these three calculations. The maximum percentage of the total source energy transformed into kinetic energy is about 17 , 


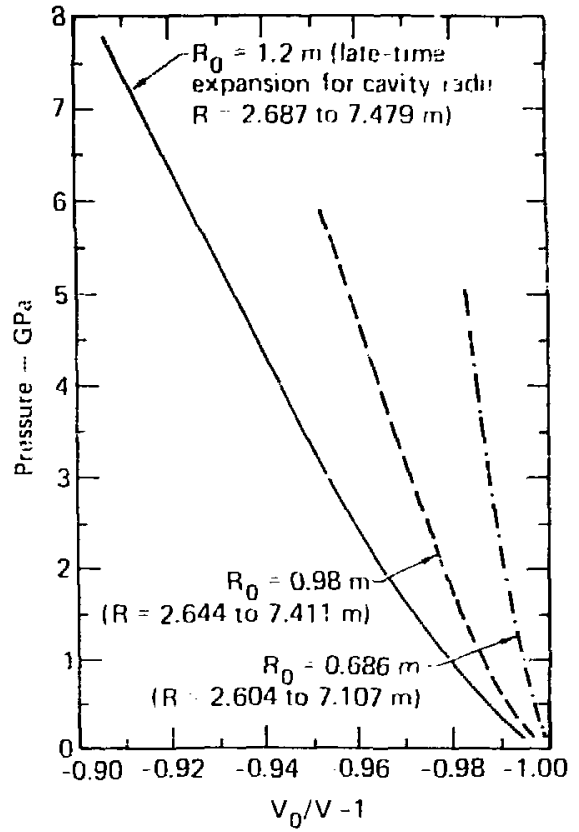

Fif. 8. Expansion of iron gas energy sourec region.

$11.2 \mathrm{~m}$ radius source), $15 \%$ (0.98 $\mathrm{m}$ radius source), or $14^{\prime \prime \prime}$ (0.686 $\mathrm{m}$ radius souree). The kinctic energy

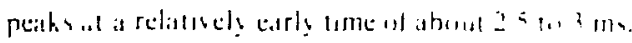

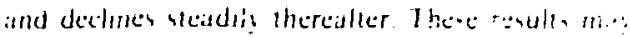

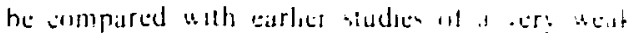

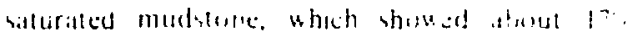

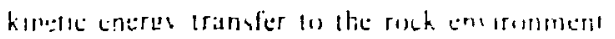

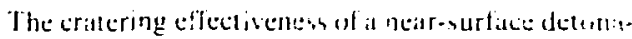

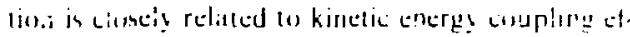
ficiency. Thus the source-sise elect reveitled here indicates that the large-radius source llaght he the

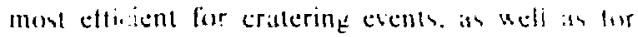
seismic encrgy eoupling al preater de;the

\section{SIRESS WAVE ATTENLATION}

The shape and allenualion of the outgenny stress wave are very nensitive ow the intrial emeres demity of the source in the reglon chate lo tote murce. Jigure 12 shows the stress profite lin three

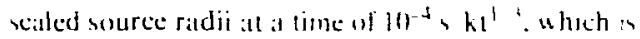
just a few microseconds after vaporiatlion of the rock has oceurred. The location of the shock wave is essentially the sanke at this lame. cren thesugt: each wave has traveled considetahly different distander from the initial source. The mignitudes af the peith stresses and their early time allenudting rale atre considerably different.

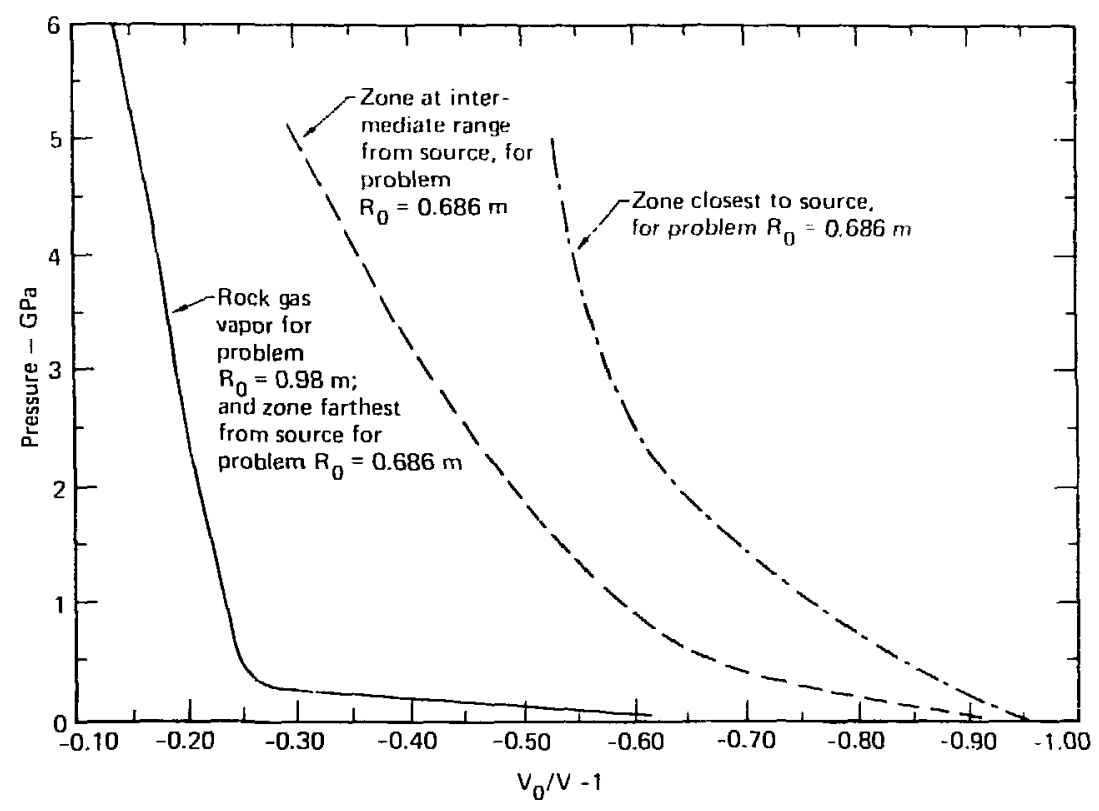

FIG. 9. Expansion of vaporized rock gas zone (average behavior); time $=0.5$ to $15 \mathrm{~ms}$. 


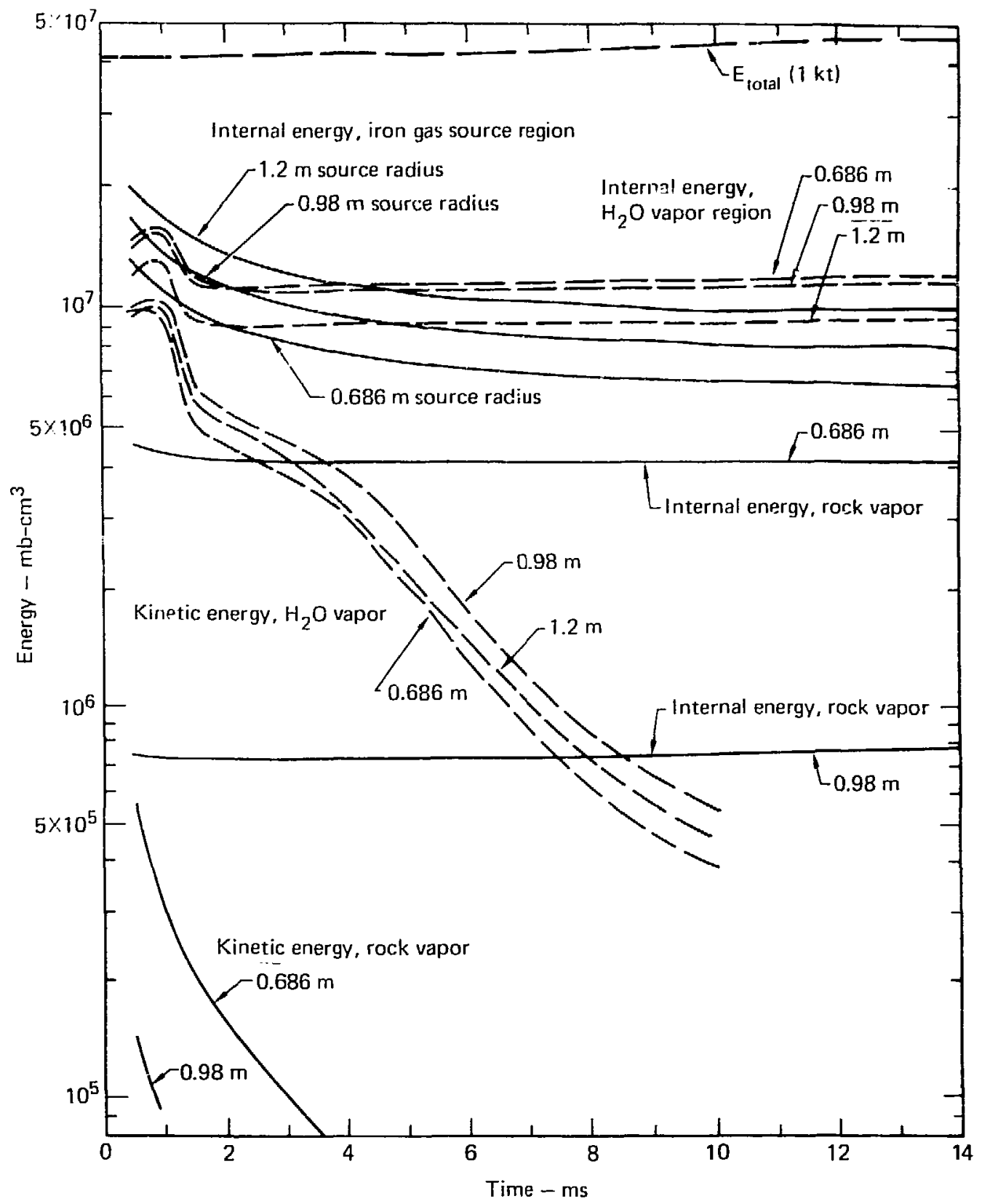

FIG. 10. Energy content of gas materials, 0.5 to $10 \mathrm{~ms}$. 


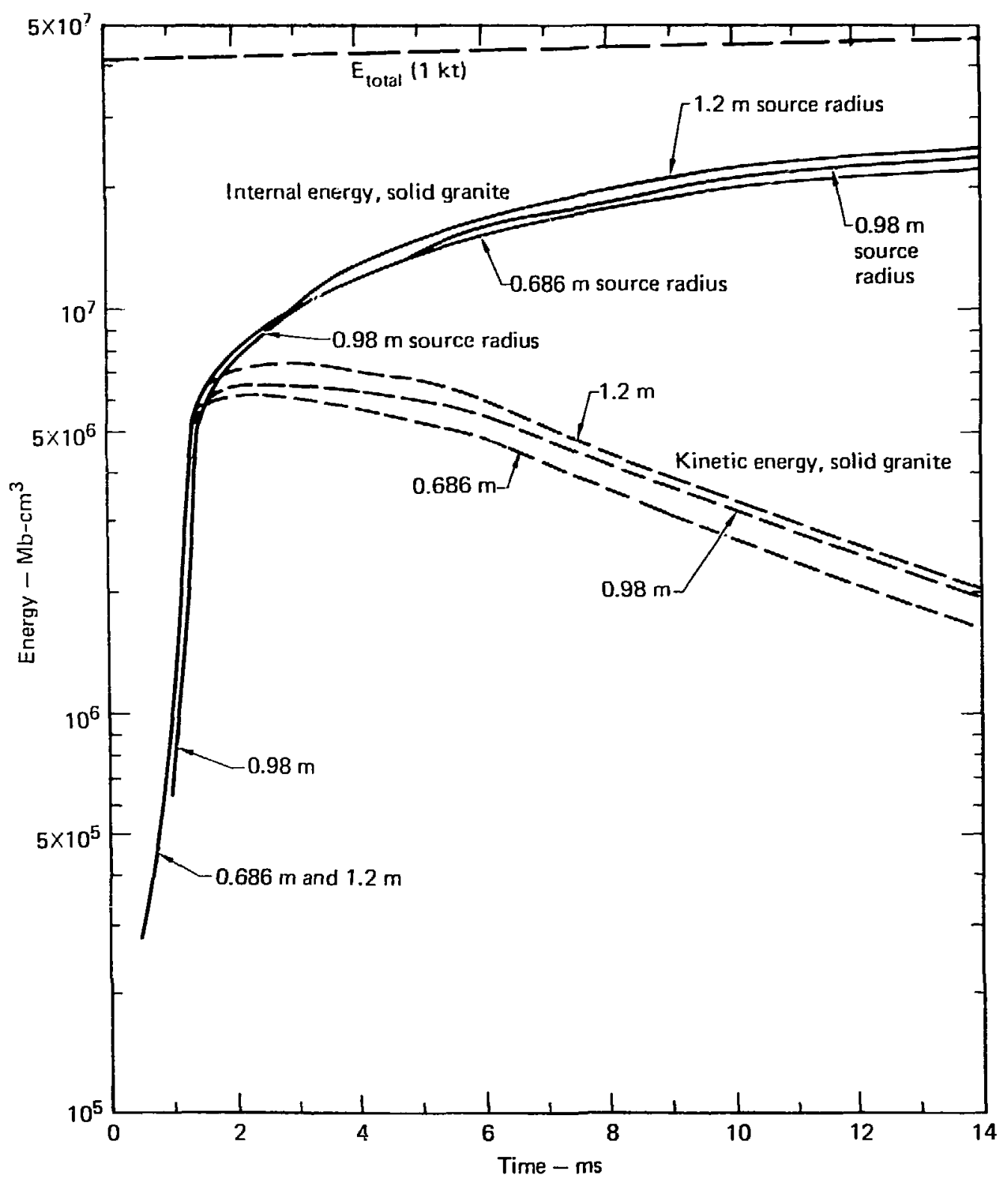

FIC. 11. Energy content in solid granite, 0.5 to 14 ms. 


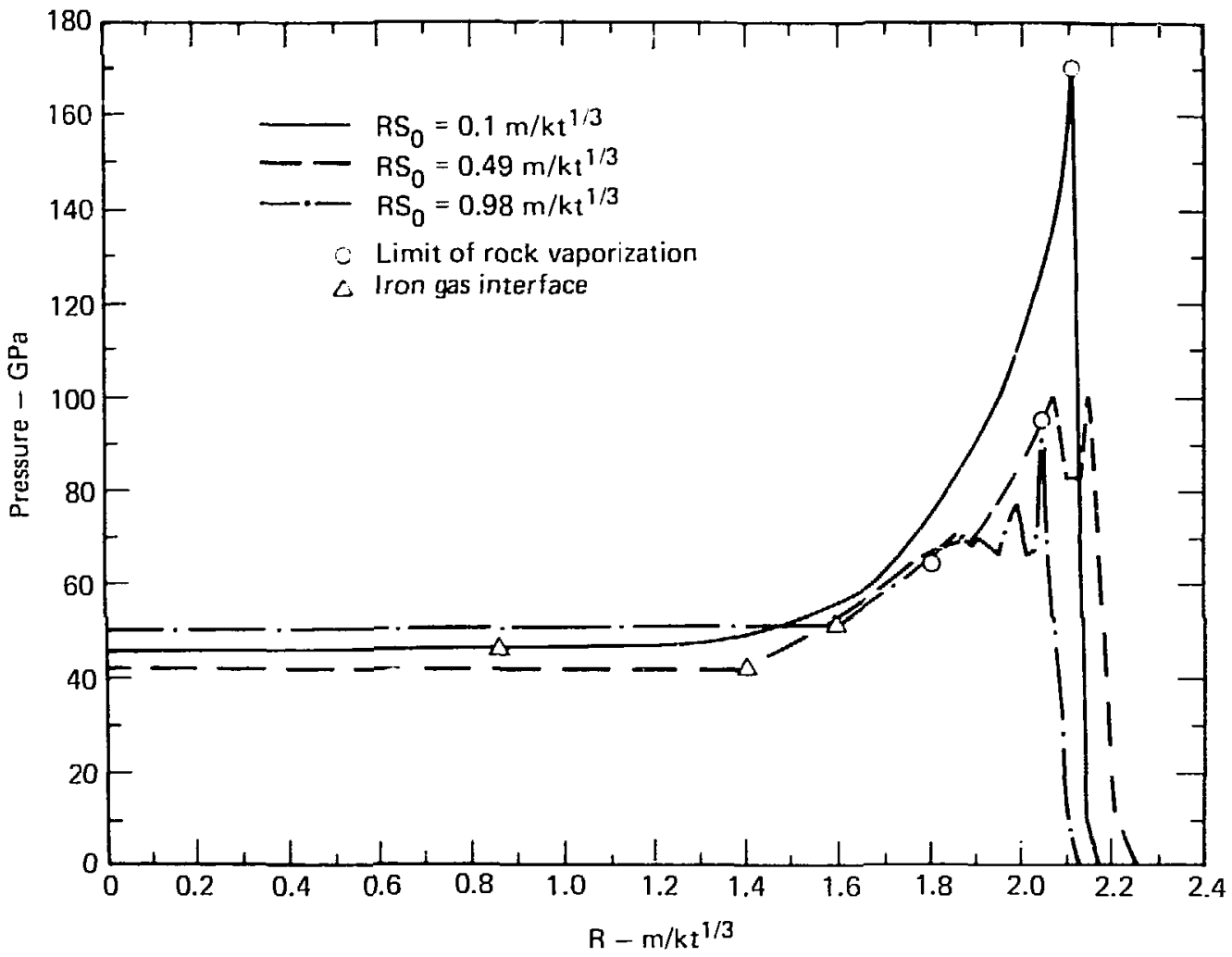

FIG. 12. Stress profile at $\mathrm{T}=10^{-4}\left(\mathrm{~s} / \mathrm{kt}^{1 / 3}\right)$.

The higher the initial energy density (the smaller the initial sealed souree radii), the greater the stress matenitude and the greater the attenuattion. The greater attenuation is a consecyuence of the larger amount of energy being deposited in the rock as was just discussed.

For those calculations that modeled a "tamiped" detonation $\left(R_{0} \leqslant 0.98 \mathrm{ml} / \mathrm{kt}^{\mathrm{l}}, 3\right)$, the magnitude and attenuation rate of the peak of the stress wave merged at ahout $+\mathrm{m} / \mathrm{kt}^{\mathrm{l}}, 3^{3}$ from the detonation center. Figure 13 shows the mignitude of the peak of the siress wave ats a function of scaled distance. Four calculations are shown where the scaled souree size varies from 0.1 to $3.0 \mathrm{~m} / \mathrm{kt}^{1}$ ? The peak stresses for scaled sources of less than or equal to $1.8 \mathrm{~m} / \mathrm{kt}^{1}{ }^{3}$ merge at a distance of $20 \mathrm{~m} / \mathrm{kt}^{1}$ ? Thus over the range of sealed source radii where enhanced coupling is calculated, the magnitude of the stress wave at fat distances from the source is in- dependent of the sealed source size. This is in agreement with previous studies. ${ }^{7}$ Only after an increase of the sealed sourec raddi beyond $2.0 \mathrm{~m} / \mathrm{kt}^{1}$ ' does the peak of the stress wave begin io reflect the partial decoupling effects.

\section{ELASTIC RESPONSE}

An examination of some of the calculated earth-motion parameters in the elastic region sielded greater insight into the sauses of enhanced seismic coupling effect. Figure 14 is a replot of the infornation shown in Fig. 4, with an enlarged linear scale $s o$ it can be compared with the following figures. Figures 15-19 show the peak stress. peak particle velocity. peat scaled displacentent, scaled final displacement, and sialed reduced displacement polential in that order. These parameters were exaluated in the clastic region as as sculed distance 


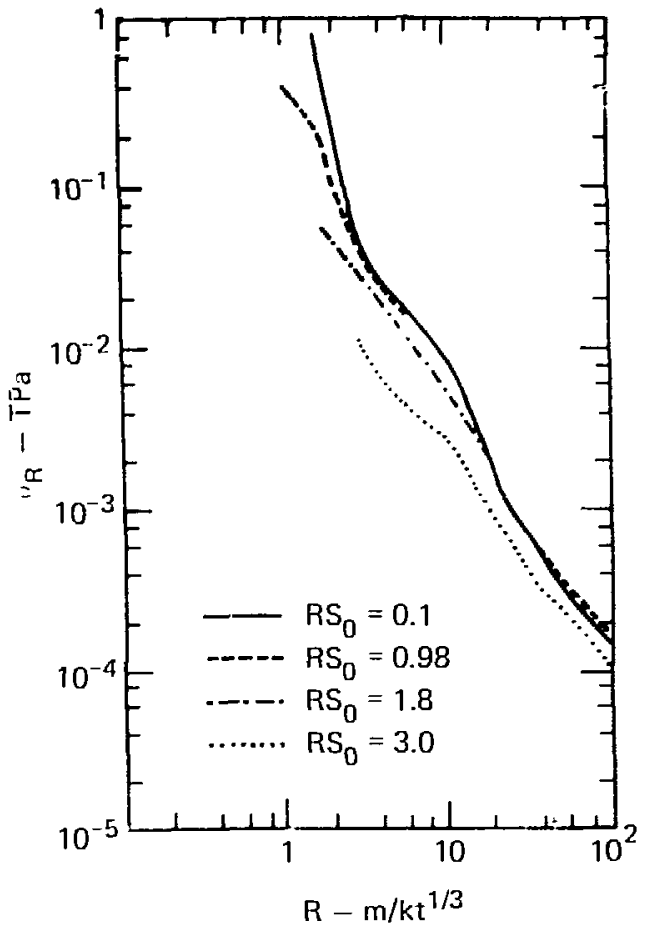

FIG. 13. Peak shock stress vs scaled range.

from the source of $100 \mathrm{~m} / \mathrm{kt}^{1} \mathrm{~s}^{3}$ for each calculation with scaled source raddii up $10+.0 \mathrm{~m} / \mathrm{kt}^{1}$ ? This covers the range of scaled souree size over which enhanced seismic coupling wis calculated.

Three observalions can be made from Figs. 14-19:

1. There is some scalter in the calculated results because of small variations in zone size.

2. There is a definite discontinuity in all the parameters in the elastic region for scaled source size between 0.78 and $0.98 \mathrm{~m} / \mathrm{kt}^{1 / 3}$. This corresponds to the drastic change in the amount of rock vaporized as the scaled radius is varied over this source size interval.

3. All parameters in the elastic region decrease rapidly for scaled source radii greater than $2.0 \mathrm{~m} / \mathrm{kt}^{1 / 3}$.

The effect of the decrease in amount of rock vaporized appears to increase all variables shown in Figs. $15-19$ by approximately $10 \%$. There is also a noticeable effect on the radius of water vaporization and final carlly radil caused by the amounl af roch. iaporided. as can be veen in fig. 14.

flegures 17 atad is thise the peish raled do placement and linal seated displatement. respeclivels. The peak displatement as esentally the integration of the partate velocts over the first positive puise and shous a suhstantially smaller increase with increased realed rourece radii than docs the final displacement. The peak particle ectecits. being essentially constant over thi interval of sealed source radii, suggests that the duration of the suress wave pulse in alse sensizive to the sealed sesurece sise and platy an important role in the enhanced seirmic cottpling shown in tig. 19.

Figure ? 0 is a plot of the seiled wavelength or lime of duration of the main pulse and rebiound pulse as the stress wate passes the point at a sealed

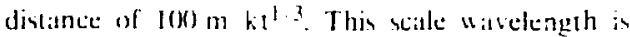
ploted against the sealed surce radii over the entire range of the parametric study. The sealed time duration of the first positive pulse is increases linearly with seitled source size up to vislues of $8.0 \mathrm{~m} \mathrm{kl}^{1}$ ? Belween $\mathrm{RS}_{11}=8.0 \mathrm{~m}(\mathrm{kt})^{1}$; and $10 \mathrm{~m} / \mathrm{kt}^{1,3}$ there is a sharp discontinuily in $\mathrm{A}$. which is probahly because there is very little displacement at the cavity boundary for catsily source sisen greater than $8.0 \mathrm{~m} / \mathrm{kt}^{\prime} 3$ (see Fig. 4).

The first negative pulse length is essentially constant for a scaled source sire, which varies from il point souree to $\mathrm{RS}_{0}=2.0 \mathrm{~m}$. $\mathrm{kl}$ ' ". then decreatsen lincitrly to $\mathrm{RS}_{0}=10 \mathrm{~m} / \mathrm{kt}^{1}$ "and remains ensenlially constant for scalled source sifes greater than $10 \mathrm{~m} / \mathrm{kt}^{1}{ }^{3}$.

Since the displatement is roughly proportional to the product of particle velocity and the duration interval, the reason for the enhanced seismic coupling elfect, apparent in Figs. 17-19, becomes clear by comparing Figs. 16 and 20 . The increase of the scaled displacement or scaled reduced displacement polential with scaled source size less than 2.0 is due primarily to the product of a constant velocity and an increasing pulse duration interval. The decrease in the energy absorbed in vaporizing or meling the rock with increasing source size occurs over a very short range of scaled source size. The energy absorbed in vaporizing the rock is approximately $10 \%$ for $\mathrm{RS}_{0} \leqslant 0.8,2 \%$ for $\mathrm{RS}_{0}=1.0$, and $0 \%$ for $\mathrm{RS}_{0} \geqslant 1.1$. The contribution to the enhanced seismic coupling effect caused by the decrease of energy absorhed in vaporization results in an approximately $7 \%$ increase in the peak particle 


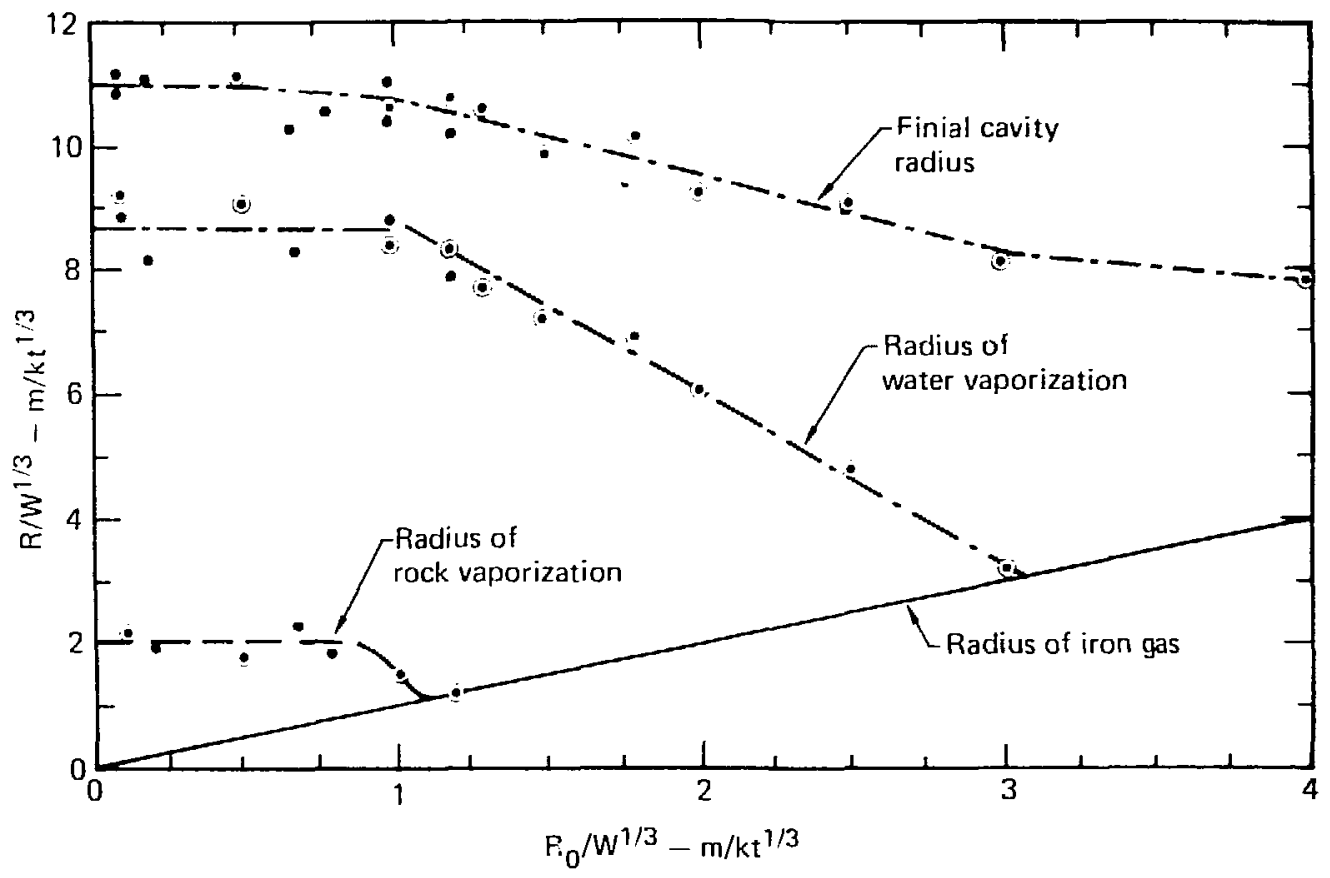

FIG. 14. Radii of major states of rock is scaled initial source size.

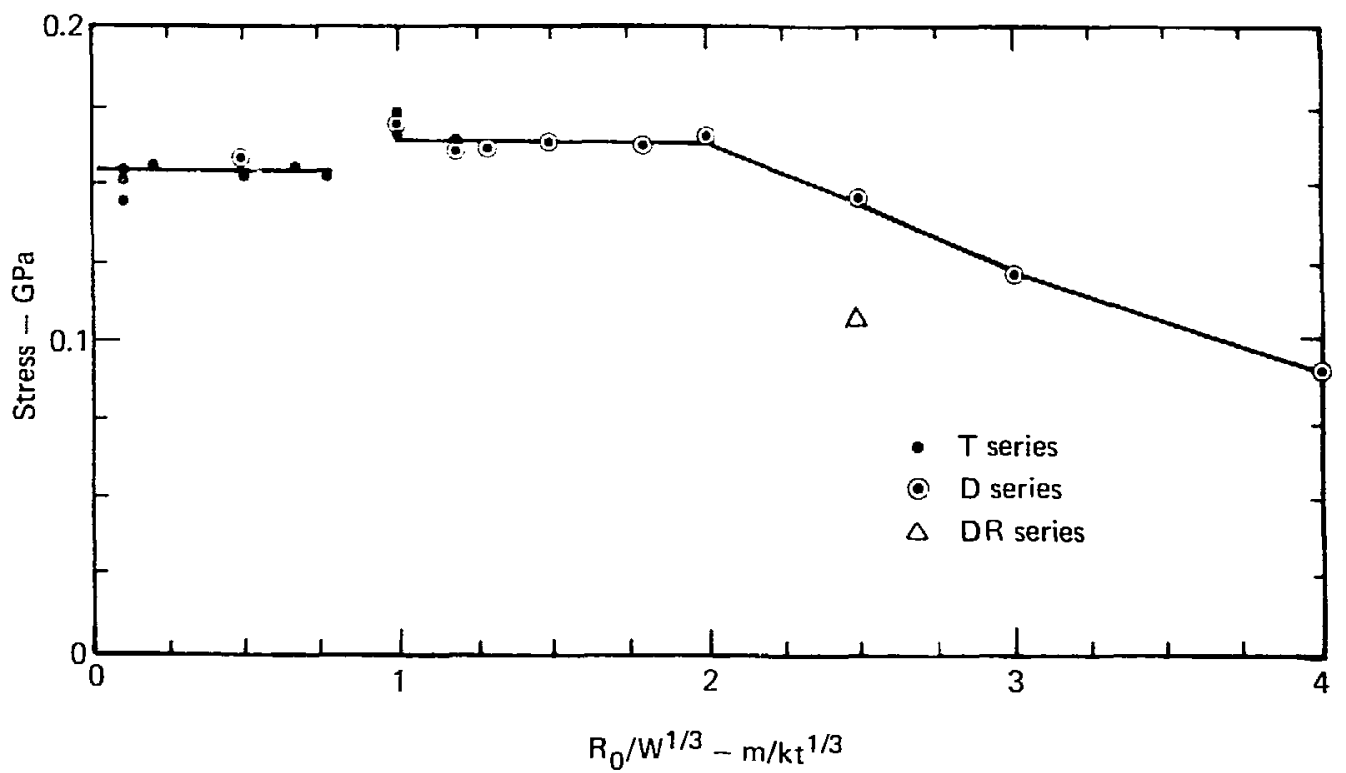

FIG. 15. Peak radial stress at scaled range of $100 \mathrm{~m} / \mathrm{kt}^{1 / 3} \mathrm{vs}$ scaled source size. 


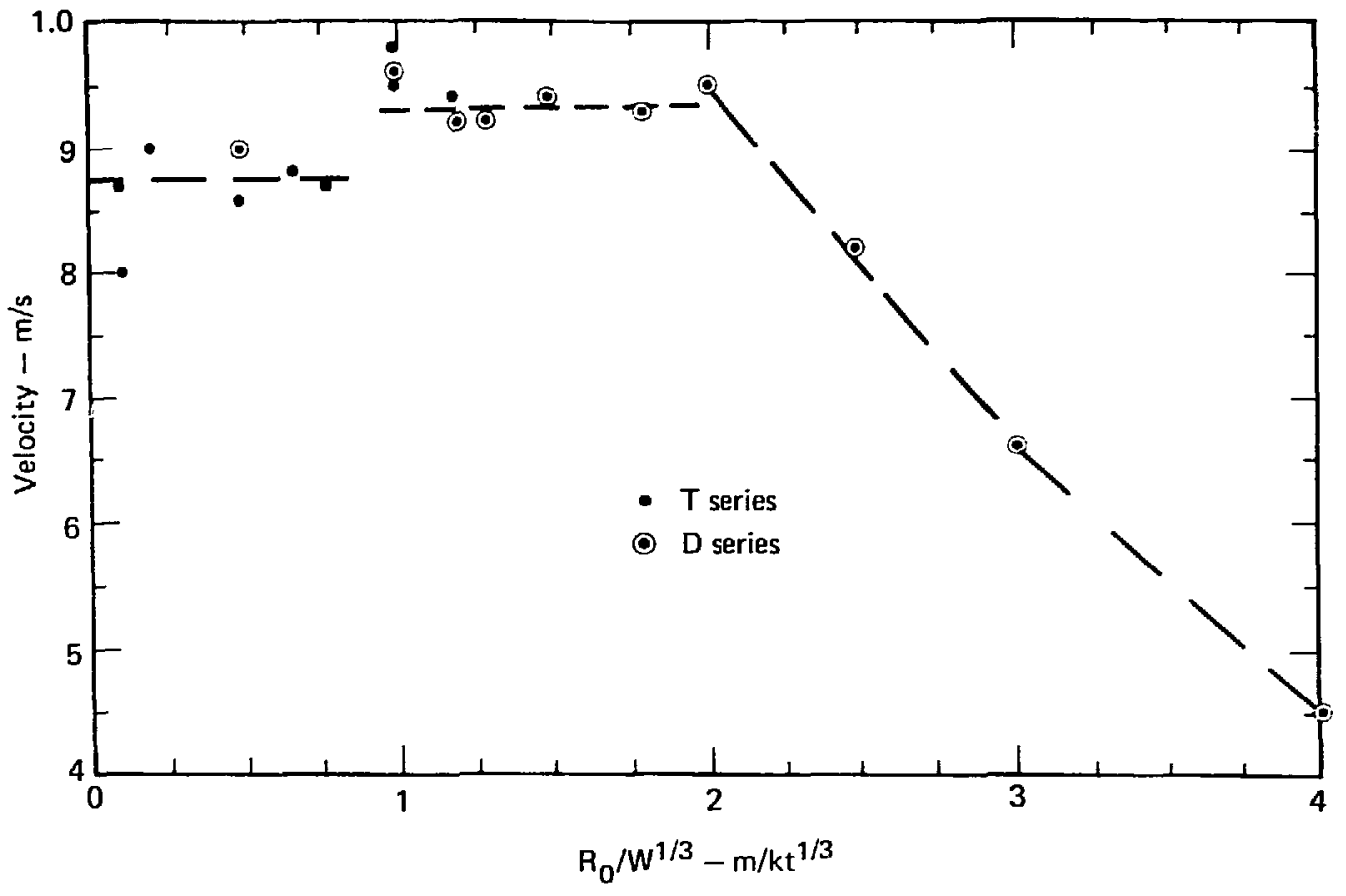

FIG. 16. Peak particle velocity at range of $100 \mathrm{~m} / \mathrm{kt}^{1 / 3} \mathrm{vs}$ scaled source radius.

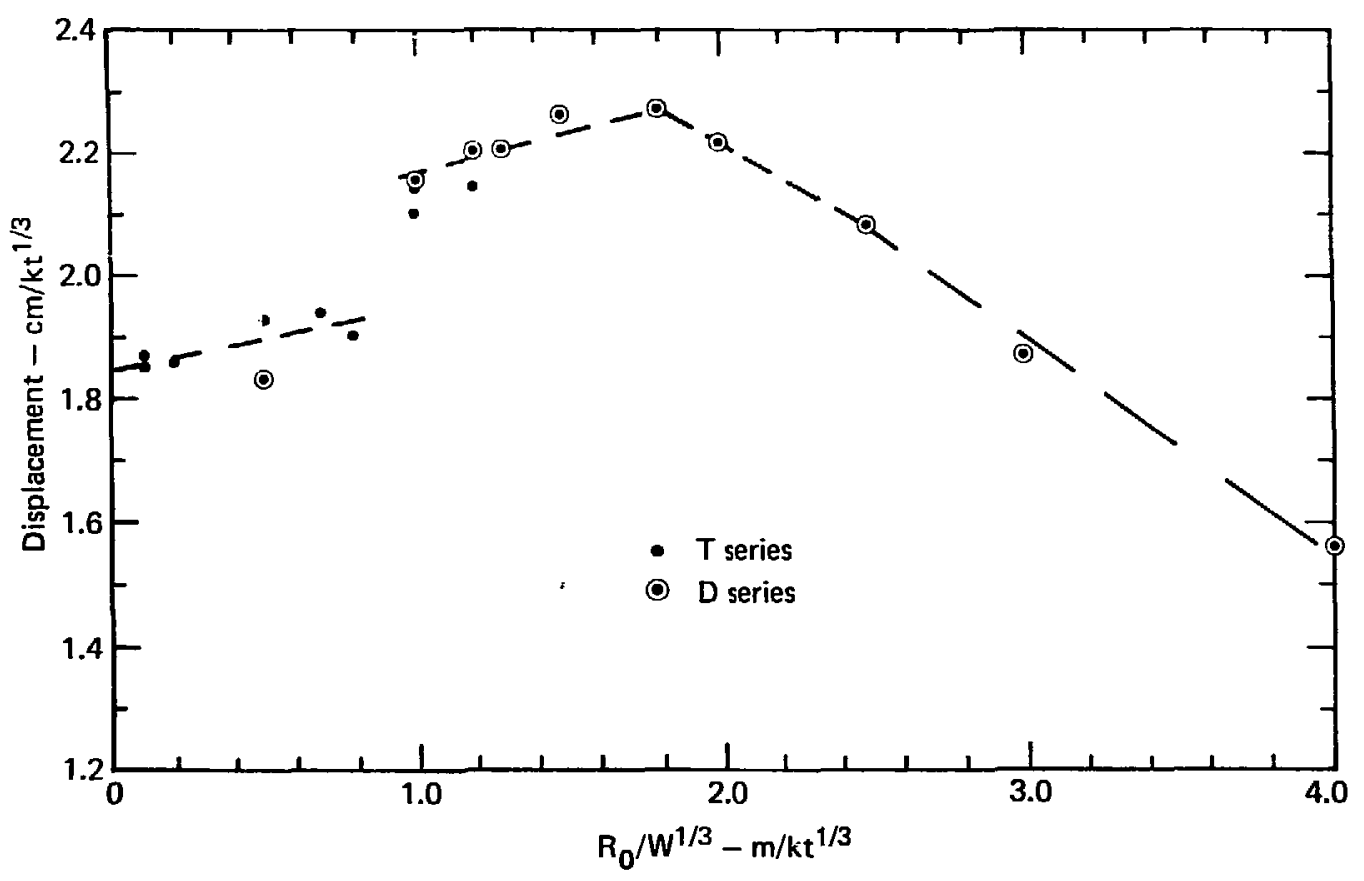

FIG. 17. Peak scaled displacement at scaled range of $100 \mathrm{~m} / \mathrm{kt}^{1 / 3} \mathrm{vs}$ scaled source radius. 


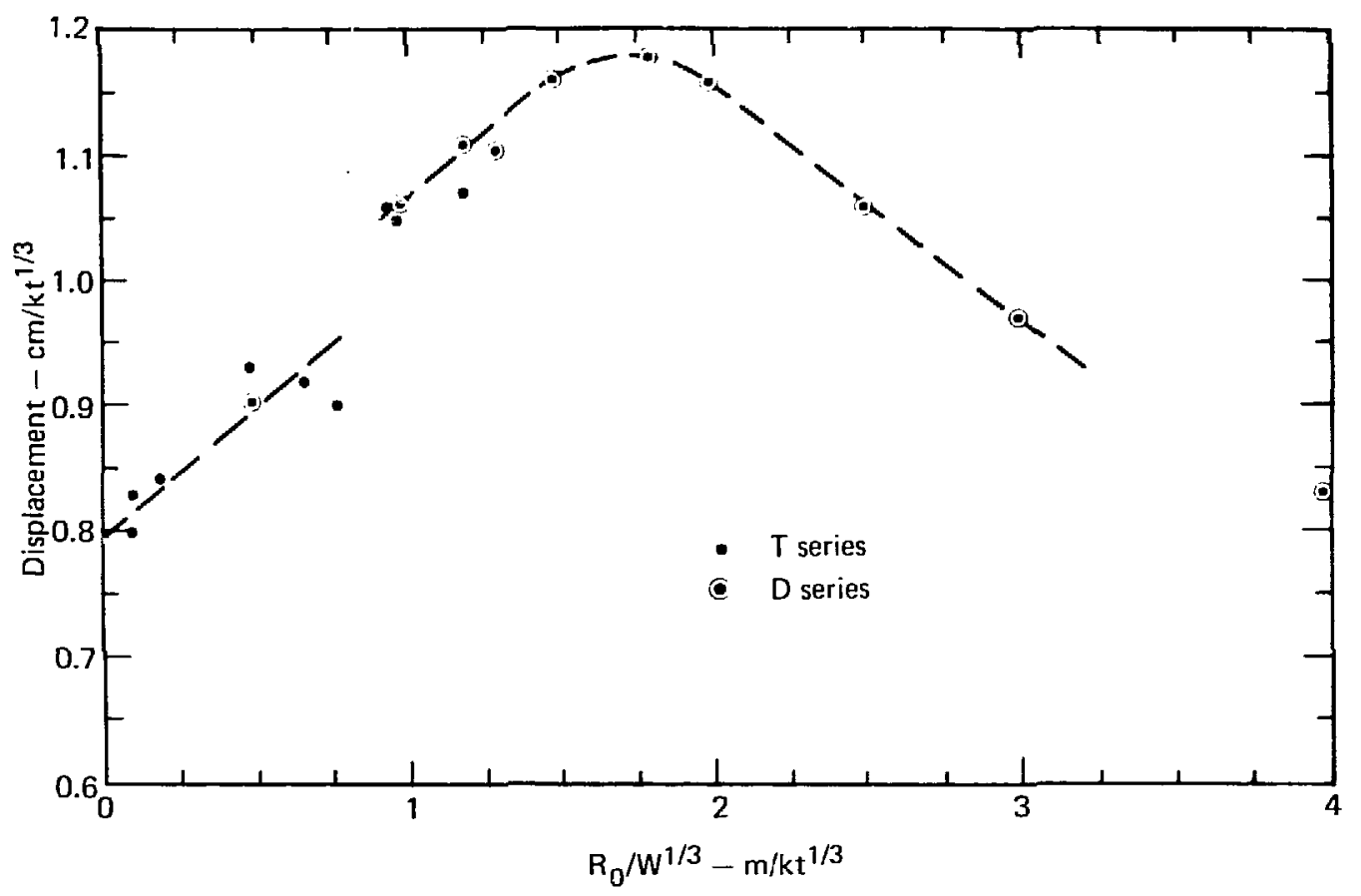

FIG. I8. Scaled final displacement at scaled range of $100 \mathrm{~m} / \mathrm{kt}^{1 / 3}$ vs scaled source radius.

velocity. The contribution of the increasing pulse duration interval to the enhanced seismic coupling effect is approximately $38 \%$ at maximum over a point source. Thus the major effect producing the snhanced scismic coupling in Fig. 19 is simply that larger sources produce longer wavelengths.

For scaled sourcc radii greater than 2.0, there is a large decrease in the peak velocity with increasing source size. Since the particle velocity decrease is greater than the increase in wave pulse duration. there is a net decrease in the displacement for increasing source radii greater than 2.0 . Thus the peak in the RDP (Figs. 2 and 19), occurring at approximately a sealed source size of $2.0 \mathrm{~m} / \mathrm{kt}^{13}$, is fully accounted for by the effect of the initial cavity size on the peak particle velocity and pulse duration. The net e.tergy available by not vaporizing the rock is only a secondary and relatively minor contribution to the enhanced RDP. Based on the simple approximation inherent in this parameler study. it appears that a detonation in a cavity with a radius of $15 \mathrm{~m} / \mathrm{kt}^{1 / 3}$ is essentially decoupled and litule is gained in going to larger cavities. 


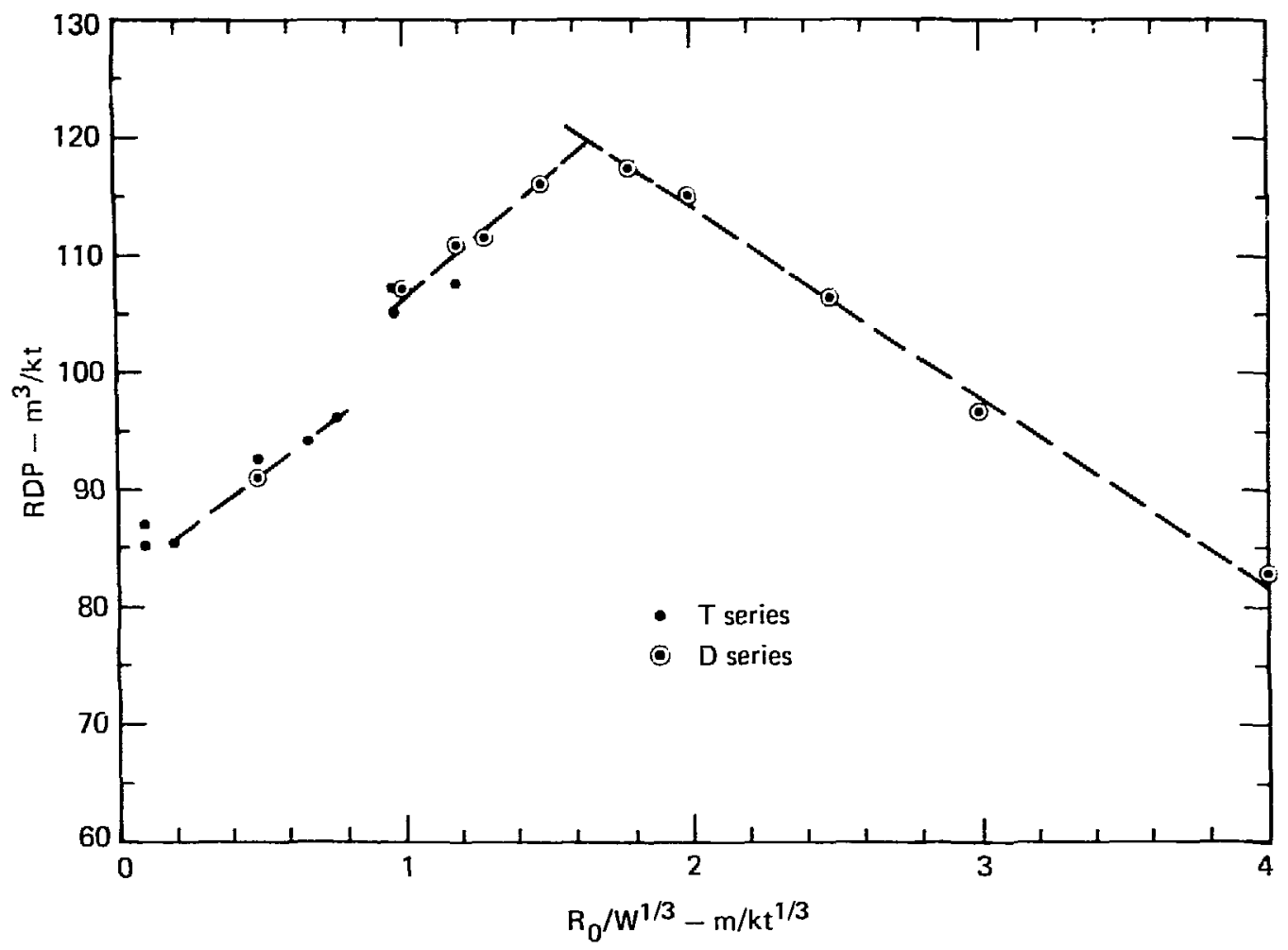

FIG. 19. Scaled reduced displacement potential vs scaled source radius.

\section{DISCUSSION WITH RESPECT TO SOME NTS NUCLEAR EVENTS}

Table 3 presents scaled emplacement data for 12 recent nuclear tests in the Pahutc Mesa, Yucca Flat, and Rainier Mesa areas at the Nevada Test Site (NTS). The scaled mass $(\mathrm{kg} / \mathrm{kt}$ ) for the Pahute Mesa and Yueca Flat explosions is based on the total mass of the canister containing the explosive. For the explosions in Rainier Mesa, the scaled mass is based on the mass of the steel walls of the shot room plus the contents of the room. The scaled radius $\left(\mathrm{m} / \mathrm{kt}^{1 / 3}\right)$ for the explosions is that for a sphere of volume equal to that of the canister (explosions 1, 2, 5-8) or shot room (explosions 3, 4, 9-12). The canisters for explosions 3 and 4 in Pahute Mesa and for explosion 9 in Yucca Flat were mplaced in larger, dir-filled cavities that had been mined at the bottom of the emplacement holes. The
TABLE 3. Explosion emplacement data.

\begin{tabular}{cccc}
\hline Arts & Event No. & $R_{0}\left(\mathrm{~m} / \mathrm{kt}^{1 / 3}\right)$ & $M(\mathrm{~kg} / \mathrm{kt})$ \\
\hline Pahute Mesa & 1 & 0.10 & 13.0 \\
& 2 & 0.11 & 4.7 \\
& 3 & 0.29 & 3.0 \\
Yucca Flat & 4 & 0.34 & 2.3 \\
& 5 & 0.36 & 171 \\
& 6 & 0.54 & 660 \\
& 7 & 0.55 & 520 \\
Rainier Mesa & 8 & 0.47 & 615 \\
& 9 & 0.86 & 18.4 \\
& 10 & 0.83 & 365 \\
& 11 & 1.12 & 780 \\
\hline
\end{tabular}




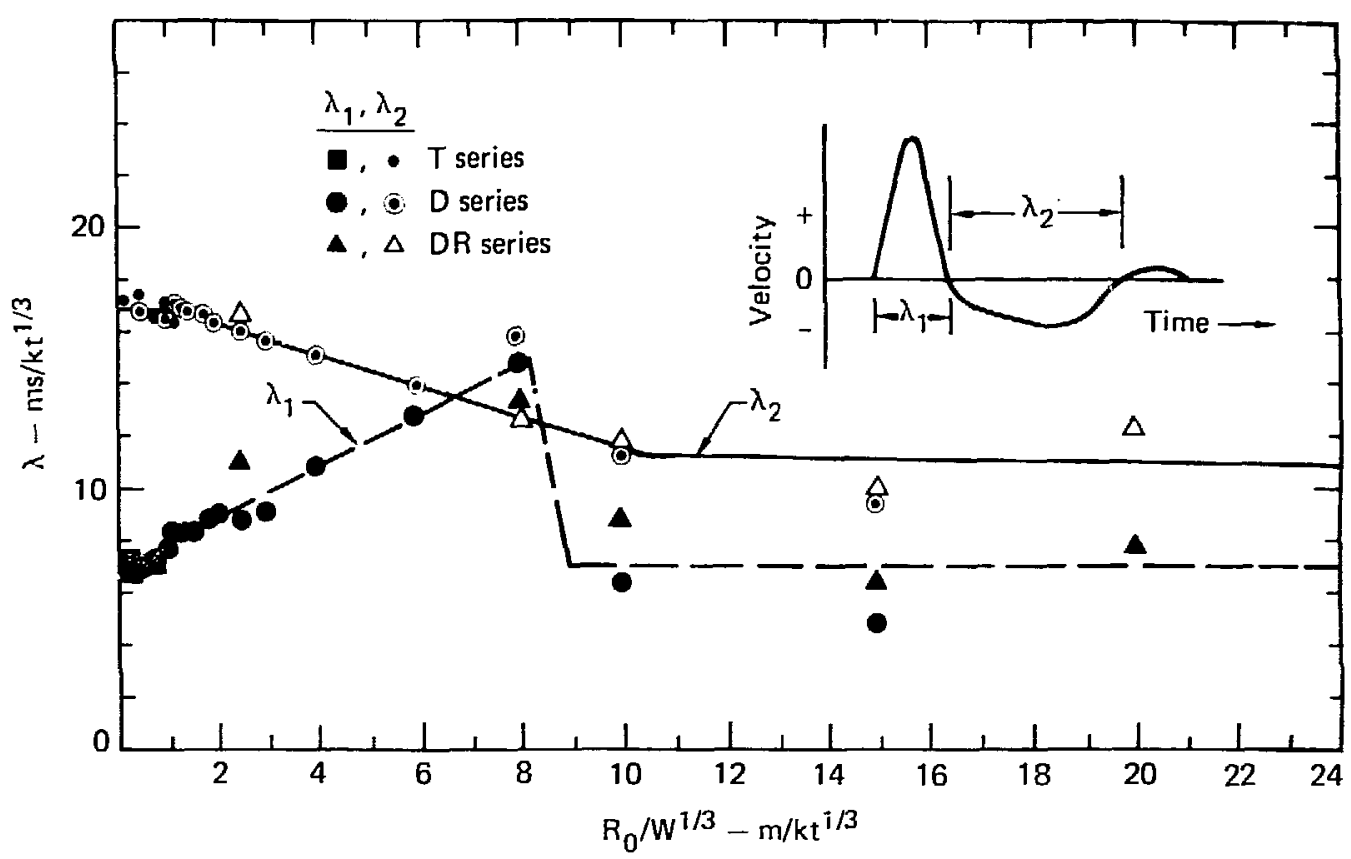

FIG. 20. Scaled wavelength vs sealed source radius.

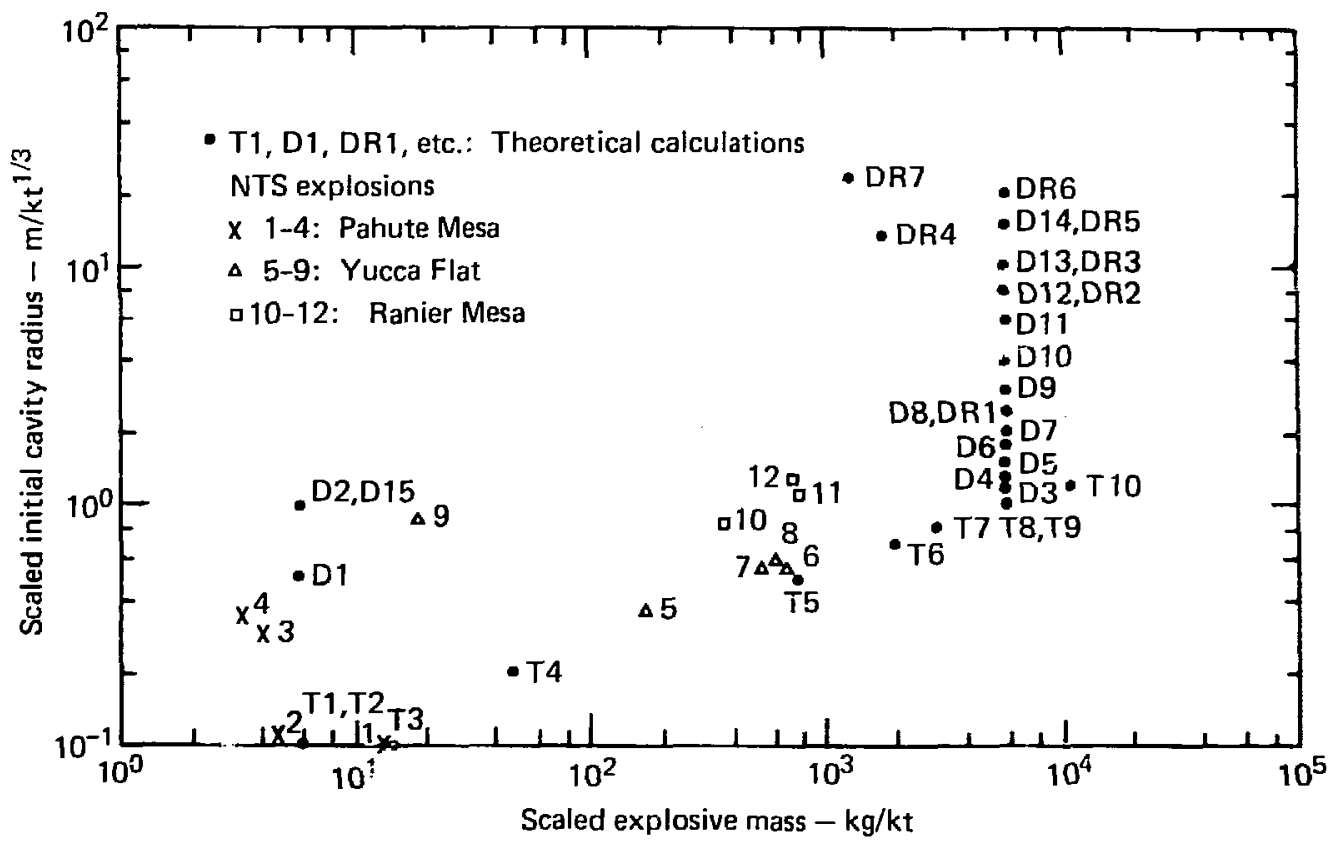

FIG. 21. Comparison of scaled explosive mass ys scaled initial cavity radius for theoretical calculations and NTS explosions. 
space between the canister and the walls of the cmplacement holes for the other explosions in Pahute Mesa and Yucea flat wis filled with stemming material (e.g., sand or peal gravel). The space between the shot-room walls and the surrounding rock in Rainic Mesa was filled with grout.

The scaled radii and acialed masses for both the NTS explosions and the calculations are plotted in Fig. 21. The NTS events fall within the same range assumed for the tamped cialculations $(0.1$ to $1.3 \mathrm{~m} / \mathrm{kt}^{1}+3$. It is also shown that the sealed radii is sealed mass relations for two of the Pahute Mesa explosions (events 1 and 2) and most Yuceil Flat explosions (events 5-8) approximate those for the constant malss that was assumed for most of the tamped calculations. The scaled mass for the two pahute Mesa explosions (events 3 and 4), one Yucea Flat explosion (event 9), and all the Rainier Mesa explosions (events 10-12), which were in mined cavities or shot rooms is less, for a given scaled radius, than that for the hypothetical explosions. We have shown in the preceding sections that, for the ranges of conditions considered, scaled radius has significantly more effect than scaled mass on seismic coupling.

Figure 19 shows the final value of scaled reduced displacement potential (RDP) as a function of the scaled energy source radius for the tamped aalculations. If the calculational results are applicable to the explosions listed in Table 3. we would expect the emplacement conditions to produce the weikest relative seismic coupling for Pahute Mesit explosions (small scaled source radii), and the strongest coupling for explosions in Rainier Mesal (near-optimum scaled source radii).

The seismic magnitude $\left(m_{b}\right)$ datal ${ }^{18}$ for NTS explosions indicate that the scismic signals (normalized to a common yield, assuming a common slope for the $m_{b}$ vs $\log _{10}$ yield relations) from
Ratinier Mesa explosions are on the atberage, at traction of a magnitude unit greater than thoxe from explosions below the water table in Yucea Flat and Pahute Mesa. This result in consisten with the sealced source radius is sealed RDP results show in in the figure. However, the qualitative agreement of these resilts does not prove that the differences in scaled radius alone are responsible for the somewhat stronger signals from Rainier Mesia explosions. Other emplacenent and geologital factor (depth, shot-point rock properties, local ges)logical structure) that affect signal generation and propadgattion could be responsible for all ar part of the observed seismic magnitude uifference.

Two of the Pahute Mesa explosions (1 and 2) have a scaled radius of about $0.1 \mathrm{~m} / \mathrm{kt}^{1.3}$ and the other two ( 3 and 4) have a sealed radius of ahout $0.3 \mathrm{~m} / \mathrm{kt}^{1 / 3}$. The results of our calculations shown in Figs. 2 and 19 indicatte that the seismic coupling of the latter explosions should be somenhat stronger. However, the magnitude residuals from the magnitude es $\log _{10}$ yield relation for Pahute Mesat, when plotted at a function of sealed radius. show no such trend for these four explosion. The scalter of the magnitude residuals for Pahute Mesa is probably determined by other variations in seismic signal generation and transmission, not by variations in scaled radius.

Similarly, the coupling of explosions 5 and $y$ in Yucea Flat might be different because of the difference in scaled radius. However, the shserved coupling for these two explosions is alnost exactly equal to the mean coupling observed below the water table in Yucca Flat.

We conclude that the above field data do not provide sufficient evidence to either support or negate the enhanced coupling that is indicated by our calculations for scaled cavity radii of 1 to $2 \mathrm{~m} / \mathrm{kl}^{1 / 3}$.

\section{ACKNOWLEDGMENTS}

We appreciate the assistance of the following people in providing emplacement data for NTS nuclear explosions: C. E. Walter, D. E. Walton, and W. Wakeman of the Lawrence Livermore Laboratory, J. W. LaComb of Defense Nuclear Agency Field Command, and C. R. Dismukes of Systems, Science and Soltware.

J. G. Trulio of Applied Theory, Incorporated, suggested this area of research and performed the first calculations demonstrating the enhanced coupling effect. He has provided useful information and comments during the course of this project.

The work discussed in this report was sponsored by the Advanced Research Projects Agency and performed under the auspices of the U.S. Department of Energy. 


\section{REFERENCES}

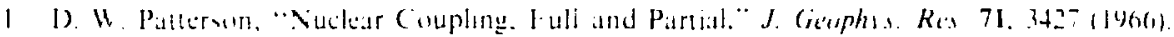

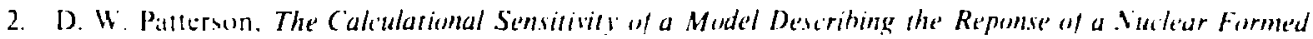
Cavity, Lawrence livermore lathoratory, livermore, (A. Rept. LCID-5125 (1966).

3. I. G. P. Seidl. SOC. A Vumerical Model for Behavior of Materials Expesed on Imense Impulsive Siresses. Laturence Livermore Lahorators. Livermort. CA. Rept. LC(D)-503? (196,5).

4. J. T. Cherrs and F. L. Petersen. "Numerical Simulation un Stress Wave Propagation from I'nderground

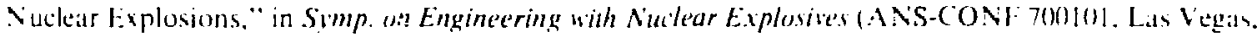
VV. January 1970). Vol. 1.

5. J. F. Schat/, SOC73. A One-Dimensional Wave Propagation Code for Roch Media. Lan rence Livermore Lahoratury, Livermore, CA, Rept. UCRL-51689 (10-2.

6. C. M. Snell. LSer' Mamual for SOC74, Lawrence Livermore Laburatory I ivermure. (A. Rept. R KCD) 76-12 (1976).

7. R. C. Schroeder, A Comparison of Intial Conditions lor Nuclear Explosion Calculations. Lasurence Livermore Laboratory. Livermure. CA. Rept, UCRL-51671 (1974).

8. W. E. Baker, P. S. Westine. and F. T. Dodge. Similarity Methods in Engineering Dinamics (Spartan Bunks. Has den Book Co.. Rucluclie Park, NJ. 1973).

9. S.L. Hancock. Numerical Comvergence os Mechanical Equilibrium with a lelocity Damping Method and its Interpretation as Simultaneous Over-relaxation, Physics Internatiunal Company. Sall Leandro. CA. Rept. TCAM 73-8 (1973).

10. T. R. Butkovich. The Gas Equation of State for Natural Materials. Liarence Livermore Liboratory. Livermore. CA, Rept. UCRL-14729 (1967).

11. T. R. Butkovich. "Calculation of the Shock Wave from an Underground Nuclear Explosion in Granite." J. Geophyr. Res. 70. 885 (1965).

12. J. T. Cherry and E. G. Rapp. Calculation of Fres-field Motion for the Piledriver Event. Laurence Livermore Laboratory, Livermure, CA. Rept. UCRL-50373 (1968).

13. J. T. Cherry and F. 1. Petersen, "Numericial Simulation of Stress Wave Propagation from Underground Nuclear Explosions," in Engincering with Nulear Explosives. CONF-700101 (American Nuclear Society, Las Vegas, NV 1970), Vol. 1.

14. J. F. Schath, Lawrence Livermore Laboratory, Livermore, CA, private communiation.

15. T. R. Butkovich, Lawrence Livermore Lahoratory, Livermore. CA, private communication.

16. R. W. Terhune. Analisis of Depth of Burial Criteria jor Containmem. Lawrence Livermure Lahoraturs. Livermore. CA. Rept. UCRL-52395 (1978).

17. D. E. Burton. C. M. Snell, and J. B. Bryan. "Computer Design of High-Eaplosise Experiments wo Simulate Subsurface Nuclear Detonations." Nucl. Tech. 26, 65 (1975).

18. R. W. Alewine III, AFTAC/VSC, private communication.

\section{$\mathrm{REH} / \mathrm{bg}$}




\section{APPENDIX A \\ INITIAL CONDITIONS}

A bbreviations used in Tables A-1 A-3

W Yield, $k ı$

$\mathrm{R}_{\text {, }} \quad$ Initial calvily radius, $m$

RM Radius of grid houndary, $m$

NC Number of zones !n souree cavity

NT Number of rones in ealculition

$Z A$ Initial zone sioe all cavity houndary, $m$

ZL Zone size al range of $100 \mathrm{~m}, \mathrm{~m}$

$\mathrm{M}_{0}$ Mass of iron for source, $\mathrm{Mg}$

$\rho_{0} \quad$ Initial density of source, $\mathrm{Mg}_{\mathrm{g}} / \mathrm{m}^{3}$

$P_{0}$ Iritial pressure of source

$\mathrm{E}_{0} \quad$ Initial energy density

$\mathrm{RS}_{0}$ Scaled source radius, $\mathrm{m} / \mathrm{kl}^{1 / 3}$

ZAS Scaled zone size ZA, m/ $/ \mathrm{kl}^{1 / 3}$

ZLS Sealed rone size $Z \mathrm{~L}, \mathrm{~m} / \mathrm{kl}^{1}$ ?

TABLE A-I. Initial conditions for tamped (T) calculations.

\begin{tabular}{|c|c|c|c|c|c|c|c|c|c|c|c|c|c|c|}
\hline & \multicolumn{14}{|c|}{ Parameters } \\
\hline & $\begin{array}{c}W \\
(k t)\end{array}$ & $\begin{array}{l}R_{0} \\
(m)\end{array}$ & $\begin{array}{l}R M \\
(m)\end{array}$ & NC & NT & $\begin{array}{l}\mathrm{ZA} \\
(\mathrm{m})\end{array}$ & $\begin{array}{l}Z L \\
(m)\end{array}$ & $\begin{array}{c}\mathrm{M}_{0} \\
(\mathrm{Mg})\end{array}$ & $\begin{array}{c}\rho_{0} \\
\left(\mathrm{Mg} / \mathrm{m}^{3}\right)\end{array}$ & $\begin{array}{c}E_{0} \\
\left(T P_{y}\right)\end{array}$ & $\begin{array}{c}\mathbf{P}_{\mathbf{0}} \\
(\mathrm{TPa})\end{array}$ & $\begin{array}{c}\text { ZAS } \\
\left(\mathrm{m} / \mathrm{kt} \mathbf{l}^{1 / 3}\right)\end{array}$ & $\begin{array}{c}\text { ZLS } \\
\left(\mathrm{m} / \mathrm{kt}^{1 / 3}\right)\end{array}$ & $\begin{array}{c}\mathrm{RS}_{0} \\
\left(\mathrm{~m} / \mathrm{kt} / / 3_{1}\right)\end{array}$ \\
\hline TI & 1000 & 0.98 & 3000 & 10 & 1000 & 0.10 & 5.2 & 5.91 & 1.5 & $1.1+3$ & $4.2+2$ & 0.01 & 0.52 & 0.1 \\
\hline $\mathbf{T} 2$ & 1000 & 0.98 & 3000 & 25 & 1015 & 0.035 & 6.4 & 5.91 & 1.5 & $1.1+3$ & $4.2+2$ & 0.0035 & 0.64 & 0.1 \\
\hline T3 & 1.0 & 0.1 & 600 & 10 & 710 & 0.011 & 0.89 & 0.014 & 3.23 & $1.1+3$ & $4.4+2$ & 0.011 & 0.89 & 0.1 \\
\hline T4 & 125 & 0.98 & 1500 & 10 & 1000 & 0.10 & 2.2 & 5.91 & 1.5 & $1 . j+2$ & $5.6+1$ & 0.02 & 0.44 & 0.2 \\
\hline T5 & 8.0 & 0.98 & 850 & 25 & 805 & 0.04 & 1.4 & 5.91 & 1.5 & 8.5 & 3.0 & 0.02 & 0.07 & 0.5 \\
\hline T6 & 1.0 & 0.686 & 600 & 7 & 788 & 0.10 & 0.52 & 2.03 & 1.5 & 3.1 & 1.1 & 0.10 & 0.52 & 0.7 \\
\hline$T 7$ & 2.0 & 0.98 & 600 & 10 & 1000 & 0.10 & 0.40 & 5.91 & 1.5 & 2.1 & 0.74 & 0.08 & 0.32 & 0.8 \\
\hline T8 & 1.0 & 0.98 & 600 & 25 & 1015 & 0.04 & 0.45 & 5.91 & 1.5 & 1.1 & 0.38 & 0.04 & 0.45 & 1.0 \\
\hline T9 & 1.0 & 0.98 & 600 & 10 & 1090 & 0.10 & 0.33 & 5.9 & 1.5 & 1.1 & 0.38 & 0.10 & 0.33 & 1.0 \\
\hline $\mathrm{T} 10$ & 1.0 & 1.2 & 600 & 12 & 793 & 0.10 & 0.51 & 10.9 & 1.5 & 0.58 & 0.21 & 0.10 & 0.51 & 1.2 \\
\hline
\end{tabular}


TABLE A-2. Initial conditions for decoupled (D) calculations.

\begin{tabular}{|c|c|c|c|c|c|c|c|c|c|c|c|c|c|c|}
\hline & \multicolumn{14}{|c|}{ Parameters } \\
\hline & $\underset{(k)}{W}$ & $\begin{array}{l}\mathrm{R}_{0} \\
(\mathrm{~m})\end{array}$ & $\begin{array}{l}R M \\
(\mathrm{~m})\end{array}$ & $\mathrm{NC}$ & NT & $\begin{array}{l}Z A \\
\text { (n) }\end{array}$ & $\begin{array}{l}Z L \\
(\mathrm{~m})\end{array}$ & $\begin{array}{c}M_{0} \\
\left(M_{g}\right)\end{array}$ & $\left(\mathrm{Mg} / \mathrm{m}^{3}\right)$ & $\begin{array}{c}\left.E_{0}\right) \\
\left(T^{\prime} P_{i}\right)\end{array}$ & $\begin{array}{c}P_{0} \\
\left(T P_{1} \cdot 1\right)\end{array}$ & $\begin{array}{c}Z \wedge S \\
\left(\mathrm{III} / \mathrm{k} \mathrm{k}^{1 / 3}\right.\end{array}$ & $\underset{\left[m_{i} / k_{1} I^{1 / 3}\right.}{\not L . S}$ & $\begin{array}{c}\mathrm{RS}_{0} \\
\left.(\mathrm{~m}) / \mathrm{kt} \mathrm{t}^{1 / 3}\right)\end{array}$ \\
\hline DI & 1000 & 4.9 & 3000 & 10 & 780 & 0.5 & 5.4 & 5.41 & $1.2-2$ & 8.5 & 2.9 & 0.05 & 0.5 & 0.5 \\
\hline $\mathrm{D} 2$ & 1000 & 9.8 & 3000 & 10 & 780 & 1.0 & 4.0 & 5.9 & $1.5-3$ & 1.1 & 0.35 & 0.1 & 0.4 & 1.11 \\
\hline D3 & 1.0 & 1.2 & 600 & 30) & 830 & 0.04 & 0.71 & 5.9 & 0.82 & 0.58 & 0.19 & 0.04 & 0.71 & 1.2 \\
\hline D4 & 1.0 & 1.3 & 600 & 13 & 813 & 0.09 & 0.59 & 5.9 & 0.64 & 0.46 & 0.15 & 0.04 & 0.54 & 1.3 \\
\hline D5 & 1.0 & 1.5 & 600 & 15 & 715 & 0.09 & 0.59 & 5.9 & 0.42 & 0.30 & $9.5-2$ & 0.09 & 0.59 & 1.5 \\
\hline D6 & 1.0 & 1.8 & 600 & is & 718 & 0.09 & 0.59 & 5.9 & 0.24 & 0.17 & $5.4-2$ & 0.09 & 0.59 & 1.8 \\
\hline D7 & 1.0 & 2.0 & 600 & 15 & 1015 & 0.12 & 0.38 & 5.9 & 0.18 & 0.13 & $3.8-2$ & 0.12 & 0.38 & 2.0 \\
\hline D8 & 1.0 & 2.5 & 600 & 25 & 725 & 0.09 & 0.58 & 5.9 & y.n-2 & $6.4-2$ & $1.9-2$ & 0.09 & 0.58 & 0.25 \\
\hline D9 & 1.0 & 3.0 & 600 & 12 & 712 & 0.22 & 0.54 & 5.4 & $5.2-2$ & $3.7-2$ & $1.1-2$ & 0.22 & 0.54 & 3.0 \\
\hline D10 & 1.0 & 4.0 & 600 & 15 & 715 & 0.24 & 0.60 & 5.9 & $2.2-2$ & $1.6-2$ & $4.3-2$ & 0.24 & 0.60 & 4.11 \\
\hline DII & 1.0 & 6.0 & 600 & 15 & 715 & 0.25 & 0.53 & 5.9 & $6.5-2$ & 4.63 & 1.2 & 0.25 & 0.60 & 6.0 \\
\hline D12 & 1.0 & 8.0 & 600 & 22 & 658 & 0.10 & 0.62 & 5.9 & $2.8-3$ & 1.95 & 0.45 & 0.10 & 0.53 & 8.0 \\
\hline D1 3 & 1.0 & 10 & 600 & 100 & 700 & 0.10 & 0.62 & 5.9 & $1.4-3$ & 1.0 & 0.22 & 0.10 & 0.62 & 10 \\
\hline $\mathrm{D} 14$ & 1.0 & 15 & 600 & 136 & 736 & 0.10 & 0.61 & 5.9 & $4.2-4$ & 0.3 & 6. $(1)-2$ & 0.10 & 0.61 & 15 \\
\hline
\end{tabular}

TABLE A-3. Initial conditions for decoupled relaxation (DR) calculations.

\begin{tabular}{|c|c|c|c|c|c|c|c|c|c|c|c|c|c|c|}
\hline & \multicolumn{14}{|c|}{ Parameters } \\
\hline & $\underset{(k \mathbf{t})}{W}$ & $\begin{array}{l}R_{0} \\
(\mathrm{~m})\end{array}$ & $\begin{array}{l}\mathrm{RM} \\
(\mathrm{m})\end{array}$ & NC & NT & $\begin{array}{l}\mathrm{ZA} \\
(\mathbf{m})\end{array}$ & $\begin{array}{l}\mathrm{ZL} \\
\text { (m) }\end{array}$ & $\begin{array}{c}\mathrm{M}_{0} \\
(\mathrm{Mg})\end{array}$ & $\stackrel{\rho_{0}}{\left(\mathrm{Mg} / \mathrm{m}^{3}\right)}$ & $\begin{array}{c}\mathrm{E}_{0} \\
(\mathrm{GPa})\end{array}$ & $\begin{array}{c}\mathbf{P}_{0} \\
(\mathbf{G P a})\end{array}$ & $\underset{\left(\mathrm{m} / \mathrm{kt} \mathrm{t}^{1 / 3}\right)}{\mathrm{ZAS}}$ & $\underset{\left(\mathrm{m} / \mathrm{kt}^{1 / 3}\right)}{\operatorname{zLS}}$ & $\underset{\left(m / k t^{1 / 3}\right)}{R S_{0}}$ \\
\hline DR 1 & 1.0 & 2.5 & 450 & 5 & 309 & 1.47 & 1.47 & 5.91 & $9.0-2$ & 6.4 & 19.0 & 1.47 & 1.47 & 2.5 \\
\hline DR2 & 1.0 & 8.0 & 450 & 10 & 310 & 1.47 & 1.47 & 5.91 & $2.8-3$ & 1.95 & 0.45 & 1.47 & 1.47 & 8.0 \\
\hline DR3 & 1.0 & 10.0 & 450 & 12 & 305 & 1.50 & 1.50 & 5.91 & $1.4-3$ & 1.0 & 0.22 & 1.50 & 1.50 & 10 \\
\hline DR4 & 1.0 & 15.0 & 450 & 15 & 305 & 1.50 & 1.50 & 5.91 & $4.2-4$ & 0.296 & 0.060 & 1.50 & 1.50 & 15 \\
\hline DR5 & 1.0 & 20.0 & 450 & 20 & 307 & 1.50 & 1.50 & 5.91 & $1.8-4$ & 0.125 & 0.025 & 1.50 & 1.50 & 20 \\
\hline DR6 $6^{a}$ & 1.0 & 13.81 & 450 & 15 & 306 & 1.50 & 1.50 & 1.82 & $1.6-4$ & 0.38 & 0.07 & 1.50 & 1.50 & 13.81 \\
\hline DR $7^{3}$ & 1.0 & 23.62 & 450 & 25 & 309 & 1.50 & 1.50 & 1.36 & $2.5-5$ & 0.076 & 0.013 & 1.50 & 1.50 & 23,62 \\
\hline
\end{tabular}

alnitial overburden stress of $18 \mathrm{MPa}$. 


\section{APPENDIX B \\ SCALED RESULTS}

Abbreviaticns used in Tables B-1-B-3

W Yitld, $k t$

$\mathrm{RS}_{0} \quad$ Suurcesize, $\mathrm{m} / \mathrm{kl}^{1 / 3}$

RRV Radius of rock vaporization, $\mathrm{m} / \mathrm{kt}^{\mathrm{j} / 3}$

RWV Radius of water vaporization, $m / k t$

RFL Radius of rock fracture limit on loading, $m / k^{1}, 3$

RFU Radius of maximum rock fracture, $m / \mathbf{k l}^{i}$ ?

$R C$ Final cavity radius, $\mathrm{m} / \mathrm{kt}^{\mathrm{l} / 3}$

$P C \quad$ Final cavity pressure, MPa

ECR Ratio of final tavity energy to yield

FRR Ratio of energy in fracturc rock to yield

ESR Ratio of seismic energy to yield

RDP Scaled reduced displacement potential, $\mathrm{m}^{3} / \mathrm{kt}$

$\lambda_{1} \quad$ Scaled positive pulse duration, $\mathrm{ms} / \mathrm{kt}^{\mathrm{l}}: 3$

$\lambda_{2}+\lambda_{\text {Scalcd total pulse duration, } \mathrm{ms} / \mathrm{kt}^{1 / 3}}$

TABLE B-1. Scaled results for tamped (T) calculations.

\begin{tabular}{|c|c|c|c|c|c|c|c|c|c|c|c|c|c|c|}
\hline & \multicolumn{14}{|c|}{ I'trameters } \\
\hline & $\begin{array}{c}W \\
(k t)\end{array}$ & $\begin{array}{c}\left.\mathrm{RS}_{(\mathrm{m} / \mathrm{kt}} 1 / 3\right) \\
\end{array}$ & $\underset{\left(\mathrm{m} / \mathrm{kt}^{\mathrm{I}} \mathrm{I} / \mathbf{3}\right)}{\operatorname{RRV}}$ & $\begin{array}{l}\text { RWV } \\
(m / k t)\end{array}$ & $\underset{\left(\mathrm{m} / \mathrm{kt} \mathbf{1}^{1 / 3}\right)}{\text { RFL }}$ & $\begin{array}{c}\text { REV } \\
(\mathrm{m} / \mathrm{kt} / / 3)\end{array}$ & $\begin{array}{c}\mathrm{RC} \\
\left(\mathrm{m} / \mathrm{kt}^{1 / 3}\right)\end{array}$ & $\begin{array}{c}\mathbf{P C} \\
\text { (MPd) }\end{array}$ & ECR & ERR & ESR & $\begin{array}{c}\text { RDP } \\
\left(m^{3} / k t\right)\end{array}$ & $n_{1}$ & $\lambda_{2}+\lambda_{1}$ \\
\hline T1 & 1000 & 0.1 & 2.03 & 9.5 & 51.6 & 77.8 & 11.2 & 48.5 & 0.534 & 0.419 & $6.0-2$ & 85.0 & 6.8 & 23.6 \\
\hline $\mathbf{T} 2$ & 1000 & 0.1 & 2.12 & 9.2 & 51.4 & 78.0 & 11.0 & 59.0 & 0.520 & 0.424 & $6.2-2$ & 87.0 & - & - \\
\hline T3 & 1.0 & 0.1 & 2.23 & 8.86 & 51.1 & 77.2 & 10.9 & 48.9 & 0.533 & 0.420 & $5.7-2$ & 85.0 & 7.2 & 24.0 \\
\hline T4 & 125 & 0.2 & 2.01 & 8.16 & 52.0 & 77.8 & 11.1 & 48.7 & 0.537 & 0.415 & $6.1-2$ & 85.2 & - & - \\
\hline T5 & 8.0 & 0.5 & 1.93 & 9.0 & 52.5 & 79.4 & 11.0 & 61.4 & 0.510 & 0.449 & $6.2-2$ & 92.5 & - & - \\
\hline T6 & 1.0 & 0.7 & 2.31 & 8.28 & 53.0 & 79.5 & 10.3 & 73.8 & 0.495 & 0.456 & $6.4-2$ & 94.0 & - & - \\
\hline $\mathrm{T} 7$ & 2.0 & 0.8 & 1.90 & 8.50 & 53.7 & 90.2 & 10.6 & 74.2 & 0.481 & 0.458 & $6.7-2$ & 96.0 & - & - \\
\hline T8 & 1.0 & 1.0 & 1.62 & 8.8 & 53.9 & 82.7 & 11.0 & 75.9 & 0.443 & 0.489 & $7.3-2$ & 105 & - & - \\
\hline$T 9$ & 1.0 & 1.0 & 1.68 & 8.4 & $55 . v$ & 82.0 & 10.4 & 75.0 & 0.426 & 0.504 & $7.7-2$ & 107 & - & - \\
\hline T10 & 1.0 & 1.2 & - & 7.9 & 53.7 & 83.2 & 10.2 & $87 . ?$ & 0.416 & 0.514 & $7.2-2$ & 107.5 & - & - \\
\hline
\end{tabular}


TABLE B-2. Scaled results for decoupled (D) calculations.

\begin{tabular}{|c|c|c|c|c|c|c|c|c|c|c|c|c|c|c|}
\hline & \multicolumn{14}{|c|}{ Parameters } \\
\hline & $\underset{(k t)}{W}$ & $\underset{(\mathrm{m} / \mathrm{kt} \mathrm{I} / 3)}{\mathrm{RS}_{0}}$ & $\begin{array}{c}\text { RRV } \\
(\mathrm{m} / \mathrm{kt} / / 3)\end{array}$ & $\begin{array}{c}\text { RWV } \\
(\mathrm{m} / \mathrm{kt})\end{array}$ & $\begin{array}{c}\text { RFL. } \\
(\mathrm{m} / \mathrm{kt} / / 3)\end{array}$ & $\begin{array}{c}\text { RFV } \\
\left(\mathrm{m} / \mathrm{kt} \mathrm{I}^{1 / 3}\right)\end{array}$ & $\begin{array}{c}\mathrm{RC} \\
(\mathrm{m} / \mathrm{kt} / / 3)\end{array}$ & $\underset{\left(M P_{1}\right)}{P C}$ & ECR & ERR & ESR & $\begin{array}{c}\text { RDP } \\
\left(\mathrm{m}^{3} / \mathrm{kt}\right)\end{array}$ & $x_{\mathbf{J}}$ & $x_{2}+x_{1}$ \\
\hline Dl & 1000 & 0.5 & 1.96 & 9.11 & 54.0 & 79.2 & 11.10 & 59.5 & 0.507 & 0.437 & $6.4-2$ & 91.0 & 7.0 & 23.8 \\
\hline D2 & 1000 & 1.0 & 1.6 & 8.4 & 54,0 & 83.4 & 10.5 & 73.6 & 0.418 & 0.506 & $7.9-2$ & 107.0 & 7.8 & 24.4 \\
\hline D3 & 1.0 & 1.2 & - & 8.3 & 53.5 & 84.3 & 10.8 & 83.3 & 0.402 & 0.525 & $7.4-2$ & 111.0 & 8.2 & 25.2 \\
\hline D4 & 1.0 & 1.3 & - & 7.7 & 55.0 & 83.8 & 80.6 & 89.6 & 0.392 & 0.535 & $7.2-2$ & 111.5 & 8.4 & 25.2 \\
\hline D5 & 1.0 & 1.5 & - & 7.2 & 54.0 & 82.0 & 9.9 & 97.0 & 0.374 & 0.554 & $7.5-2$ & 116.0 & 8.4 & 25.0 \\
\hline D6 & 1.0 & 1.8 & - & 6.9 & 53.0 & 84.7 & 10.2 & 113 & 0.353 & 0.573 & $7.5-2$ & 117.5 & 8.8 & 25.4 \\
\hline D7 & 1.0 & 2.0 & - & 6.12 & 53.0 & 82.0 & 9.3 & 125 & 0.357 & 0.569 & $7.5-2$ & 115.0 & 9.0 & 25.2 \\
\hline DB & 1.0 & 2.5 & - & 4.78 & 51.0 & 81.9 & 9.1 & 168 & 0.396 & 0.541 & $6.3-2$ & 106.5 & 8.8 & 24.8 \\
\hline D9 & 1.0 & 3.0 & - & 3.22 & 42.0 & 78.5 & 8.1 & 215 & 0.463 & 0.790 & $4.9-2$ & 97.0 & 9.2 & 24.8 \\
\hline DIO & 1.0 & 4.0 & - & - & 39.0 & 72.5 & 7.85 & 300 & 0.597 & 0.375 & $3.0-2$ & 83.0 & 10.8 & 25.8 \\
\hline D11 & 1.0 & 6.0 & - & - & 20 & 57.0 & 7.7 & 420 & 0.831 & 0.159 & $1.1-2$ & 49.5 & - & - \\
\hline D12 & 1.0 & 8.0 & - & - & 18 & 40.6 & 8.6 & 362 & 0.962 & $3.5-2$ & $3.6-3$ & 19.4 & - & - \\
\hline D13 & 1.0 & 10.0 & - & - & 13.6 & 27.9 & 10.2 & 210 & 0.994 & $4.6-3$ & $1.2-3$ & 6.2 & - & - \\
\hline D1 4 & 1.0 & 15 & - & - & - & 16.4 & I5.1 & 60.3 & 0.9998 & $1.8-5$ & $2.1-4$ & 1.2 & - & - \\
\hline
\end{tabular}

TA BLE B-3. Scaled results for decoupled relaxed (DR) calculations.

\begin{tabular}{|c|c|c|c|c|c|c|c|c|c|c|c|c|c|c|}
\hline & \multicolumn{14}{|c|}{ Parameters } \\
\hline & $\begin{array}{c}W \\
\text { (kt) }\end{array}$ & $\begin{array}{c}\mathrm{RS}_{0} \\
\left(\mathrm{~m} / \mathrm{kt}^{\mathrm{I}} \mathrm{l} / \mathbf{3}\right)\end{array}$ & $\begin{array}{c}\text { RRV } \\
\left(\mathrm{m} / \mathrm{kt}^{\mathrm{l}} / \mathbf{3}\right)\end{array}$ & $\begin{array}{c}\text { RWV } \\
(m / k t)\end{array}$ & $\begin{array}{c}\text { RFL } \\
(\mathrm{m} / \mathrm{kt} \mathrm{I} / \mathbf{3})\end{array}$ & $\underset{\left(m / k t^{1 / 3}\right)}{\text { RFV }}$ & $\begin{array}{c}\mathrm{RC} \\
\left(\mathrm{m} / \mathrm{kt}^{1 / 3}\right)\end{array}$ & $\begin{array}{c}\mathrm{PC} \\
(\mathrm{MPa})\end{array}$ & ECR & EF.R & ESR & $\begin{array}{c}\text { RDP } \\
\left(m^{3} / k t\right)\end{array}$ & $\lambda_{1}$ & $\lambda_{2}+\lambda_{1}$ \\
\hline DRI & 1.0 & 2.5 & - & 5.44 & 51 & 83.5 & 9.7 & 157 & 0.405 & $0.5 \leqslant 0$ & $4.8-2$ & 111.0 & 11.0 & 27.6 \\
\hline DR 2 & 1.0 & 8.0 & - & - & 18 & 43.4 & 9.8 & 355 & 0.959 & $4.0-2$ & $3.4-2$ & 21.2 & 12.8 & 26.0 \\
\hline DR3 & 1.0 & 10 & - & - & 19.0 & 31.0 & 11.6 & 208 & 0.993 & $6.2-3$ & $1.3-3$ & 7.5 & 8.8 & 20.4 \\
\hline DR4 & 1.0 & 15 & - & - & - & 19.5 & 16.5 & 60.2 & 0.9995 & 5.3-5 & 8.9-5 & 2.4 & 6.4 & 16.2 \\
\hline DR5 & 1.0 & 20 & - & - & - & 23.0 & 21.5 & 24.7 & 1.0 & - & - & 2.07 & 7.8 & 20.2 \\
\hline $\operatorname{DR}^{\mathrm{a}}$ & 1.0 & 13.81 & - & - & - & 24.3 & 15.3 & 69.0 & 0.9993 & $3.1-4$ & $2.7-4$ & 2.7 & 6.8 & 17.2 \\
\hline $\operatorname{DR} 7^{a}$ & 1.0 & 23.62 & - & - & - & - & 25.1 & 13.1 & 1.0 & - & - & 1.8 & 8.4 & 23.0 \\
\hline
\end{tabular}

Initial overburden stress of $18 \mathrm{MFa}$. 